IZA DP No. 7457

Partial Identification of the Long-Run Causal Effect of Food Security on Child Health

Daniel L. Millimet

Manan Roy

June 2013 


\title{
Partial Identification of the Long-Run Causal Effect of Food Security on Child Health
}

\author{
Daniel L. Millimet \\ Southern Methodist University \\ and IZA \\ Manan Roy \\ IMPAQ International, LLC \\ Discussion Paper No. 7457 \\ June 2013 \\ IZA \\ P.O. Box 7240 \\ 53072 Bonn \\ Germany \\ Phone: +49-228-3894-0 \\ Fax: +49-228-3894-180 \\ E-mail: iza@iza.org
}

Any opinions expressed here are those of the author(s) and not those of IZA. Research published in this series may include views on policy, but the institute itself takes no institutional policy positions. The IZA research network is committed to the IZA Guiding Principles of Research Integrity.

The Institute for the Study of Labor (IZA) in Bonn is a local and virtual international research center and a place of communication between science, politics and business. IZA is an independent nonprofit organization supported by Deutsche Post Foundation. The center is associated with the University of Bonn and offers a stimulating research environment through its international network, workshops and conferences, data service, project support, research visits and doctoral program. IZA engages in (i) original and internationally competitive research in all fields of labor economics, (ii) development of policy concepts, and (iii) dissemination of research results and concepts to the interested public.

IZA Discussion Papers often represent preliminary work and are circulated to encourage discussion. Citation of such a paper should account for its provisional character. A revised version may be available directly from the author. 


\section{ABSTRACT}

\section{Partial Identification of the Long-Run Causal Effect of Food Security on Child Health}

Food security and obesity represent two of the most significant public health issues. However, little is known about how these issues are intertwined. Here, we assess the causal relationship between food security during early childhood and relatively long-run measures of child health. Identifying this causal relationship is complicated due to endogenous selection and misclassification errors. To overcome these difficulties, we utilize a nonparametric bounds approach along with data from the ECLS-K and ECLS-B. The analysis reveals a positive association between food insecurity and future child obesity in the absence of misclassification. However, under relatively innocuous assumptions concerning the selection process, we often obtain bounds that indicate a negative causal effect of food insecurity on future child obesity. All results are extremely sensitive to misclassification.

JEL Classification: $\quad$ C14, C21, I12, I32

Keywords: food insecurity, health outcomes, nonclassical measurement error, nonparametric bounds

Corresponding author:

Daniel L. Millimet

Department of Economics

Southern Methodist University

Box 0496

Dallas, TX 75275-0496

USA

E-mail: millimet@smu.edu 


\section{Introduction}

The U.S. currently confronts two significant issues related to the health and nutrition of its population. The first is food insecurity. The second is obesity. Food insecurity is a metric of material hardship designed to measure 'hunger' in the U.S. (Bhattacharya et al. 2004). For households with children, it is measured using the 18-question Core Food Security Module (CFSM), with a greater number of affirmative responses indicating greater levels of food insecurity. Households responding in the affirmative to at least one question are categorized as marginally food secure; affirmative responses to at least three (eight) questions are categorized as low (very low) food secure. Obesity, either as a child or adult, is defined as having a body mass index (BMI) above the $95^{\text {th }}$ percentile of the age- and gender-specific reference population. Overweight is defined as above the $85^{\text {th }}$ percentile of the same distribution.

The twin issues of food insecurity and obesity have received significant attention of late. In terms of food insecurity, the most recent figures indicate that $14.9 \%$, or 17.9 million, households were food insecure in $2011 .{ }^{1}$ Of these, $9.2 \%$, or 11.0 million, households were classified as low food secure; $5.7 \%$, or 6.8 million, were classified as very low food secure. Among households with children under the age of 18, 10.6\%, or 4.1 million, had one or more food insecure adults (but only food secure children); 10.0\%, or 3.9 million, contained both adults and children classified as food insecure. These figures represent a sizeable increase from 1995, attributable mainly to the Great Recession. See Coleman-Jensen et al. (2012) for further details.

Food insecurity is associated with a host of child health problems (Gundersen 2013; Gundersen et al. 2011; Van den Berg et al. 2011). Some of the health problems associated with food insecurity are: greater cognitive problems (Howard 2011); higher levels of aggression and anxiety (Whitaker et al. 2006); higher probability of being anemic (Eicher-Miller et al. 2009); higher probabilities of being hospitalized (Cook et al. 2006); lower nutrient intakes (Cook et al. 2004); poorer general health (Cook et al. 2006); higher probabilities of dysthymia and other mental health issues (Alaimo et al. 2002); higher probabilities of asthma (Kirpatrick et al. 2010); higher probabilities of behavioral problems (Huang et al. 2010); and more instances of oral health problems (Muirhead et al. 2009). In fact, one of the primary reasons for the increasing interest in food insecurity is its potential association with nutritional deprivation (Bhattacharya et al. 2004). In sum, Gundersen et al. (2011, p. 282) characterize food insecurity as "one of the most important and high profile nutrition-related public health issues in the United States today."

In terms of obesity, and child obesity in particular, the prevalence of obese adolescents has tripled in the last thirty years; it has more than doubled for younger children. Specifically, the rate of child obesity

\footnotetext{
${ }^{1}$ See http://www.ers.usda.gov/topics/food-nutrition-assistance/food-security-in-the-us/key-statistics-graphics.aspx.
} 
increased from $5 \%$ to $10.4 \%$ for $2-5$ year old children, from $4.0 \%$ to $19.6 \%$, and from $6.1 \%$ to $18.1 \%$ for 12 to 19 year-olds between 1971 and 2008 (Ogden and Carroll 2010). While obesity is a concern for children from all demographic groups, its greater prevalence within lower socioeconomic populations is well established (e.g., Rosin 2008; Liping et al. 2012). In 2010, 2.1\% of pre-school children from low income families were extremely obese while $15.0 \%$ of low-income pre-school children were obese (Liping et al. 2012). Brisbois et al. (2012, p. 347) state: "Obesity is considered to be a worldwide epidemic with little evidence that its incidence is declining or that it has even reached a plateau."

As childhood obesity has received greater attention, its consequences have becoming increasingly welldocumented. Obesity burdens individuals with severe physical, economic, and emotional suffering, and puts children and adolescents at risk for a number of health problems such as those affecting cardiovascular health, the endocrine system, and mental health (Deckelbaum and Williams 2001). Dietz and Gortmaker (2001) note that $60 \%$ of overweight children aged five to ten years old have at least one associated cardiovascular disease risk factor. Moreover, obesity is persistent; childhood obesity is highly correlated with adolescent and adult obesity (Serdula et al. 1993; Liping et al. 2012). In the U.S., the total cost attributable to obesity was over $\$ 75$ billion in 2000 according to Finkelstein et al. (2004). More recent estimates put the cost over $\$ 200$ billion (Cawley and Meyerhoefer 2012). Walpole et al. (2012) calculate that North America accounts for $34 \%$ of the total human biomass in the world despite containing only $6 \%$ of the world population. Moreover, the authors estimate that if the entire world had the same BMI distribution as the U.S., this would be equivalent to an additional 935 million people in the world of average BMI. Based on a U.S. poll in 2008, obesity tops the list of health problems children face (Cawley 2010). Globally, the World Health Organization ranks obesity among the top ten global public health issues (WHO 1998).

As is evident, food insecurity and obesity represent two significant public health issues in the U.S. However, research related to these problems has remained predominantly distinct. This is, perhaps, not surprising given that 'hunger' and 'obesity' are not usually seen as related. While this may be true in the short-run, the long-run relationship between food insecurity and child BMI is less clear. In this paper, we address this issue by assessing the causal effect of food insecurity on long-run child obesity and overweight status.

The long-run relationship between household food insecurity and child BMI is complex, being potentially affected by a number of a factors. First, because food insecurity directly impacts the quantity and quality of food available in the household, nutritional habits may be altered even after a household is elevated to being food secure. For example, households may inadvertently develop unhealthy consumption patterns such as overeating when food is available or consuming energy-dense foods that have no nutri- 
tional value (Kuku et al. 2012). Such unhealthy eating patterns may be exacerbated or mitigated over time through participation in nutrition assistance programs such as the School Breakfast Program (SBP), National School Lunch Program (NSLP), and Supplemental Nutrition Assistance Program (SNAP). ${ }^{2}$ Second, there is increasing evidence that nutrition in utero and during early infancy have long-run consequences on obesity (Dietz 1997; Martorell et al. 2001). Thus, undernutrition due to food insecurity, particularly at critical junctures of fetal, infant, and child development may have long-term effects on future obesity status.

Existing research sheds little light on the long-run causal relationship between food insecurity and child BMI for two reasons. First, the majority of existing studies focus purely on association, not causation. The reported associations run the gamut, finding either no relationship (e.g. Bhattacharya et al. 2004; Martin and Ferris 2007; Bhargava et al. 2008; Gundersen et al. 2008, 2009), a negative relationship (e.g., Matheson et al. 2002; Jimenez-Cruz et al. 2003; Rose and Bodor 2006), or even a positive correlation (e.g., Jyoti et al. 2005; Casey et al. 2006; Dubois et al. 2006). Second, the majority of existing studies focus on contemporaneous associations between food insecurity and child outcomes. Moreover, existing studies that do attempt to identify the causal effect of food insecurity on child BMI also focus on contemporaneous effects. As such, Martorell et al. (2001, p. 878S) conclude that "the evidence linking undernutrition to future risk of fatness is limited and contradictory."

As stated above, in this paper we wish to move beyond associations and assess the causal effect of food insecurity on future child obesity and overweight status. To do so, requires us to address two identification issues. First, food insecure households do not constitute a random sample of the population. Observed (in the data) characteristics and unobserved attributes of households may be associated both with a higher propensity to be food insecure and a higher propensity of obesity among its child members. Second, food insecurity status is often mismeasured or misreported in household surveys. People may misreport food insecurity status (e.g., Hamelin et al. 2002) or it may be mismeasured (Gundersen and Kreider 2008, 2009). This study extends the literature on the long-term consequences of food insecurity on childhood obesity and child overweight by accounting for non-randomness and mismeasurement of food security status in household survey data.

\footnotetext{
${ }^{2}$ In response to concerns about food insecurity, many nutrition assistance programs exist in the U.S., with SNAP being the largest (Coleman-Jensen et al. 2012). Gundersen and Kreider (2011) find a beneficial, causal effect of SNAP participation on food security after accounting for non-random selection and measurement error in reports of both SNAP participation and food insecurity. Schanzenbach (2009) and Millimet et al. (2010) find a detrimental, causal effect of NSLP participation on child obesity. Millimet et al. (2010, 2012) obtain a beneficial, causal effect of SBP participation on child obesity. Private food assistance programs administered through the nationwide network of Feeding America are additional sources of food assistance for families (Fiese et al. 2011).
} 
To proceed, we begin by utilizing panel data on over 6,400 children from relatively low socioeconomic status (SES) households during early primary school obtained from the Early Childhood Longitudinal Survey - Kindergarten Class of 1998-99 (ECLS-K). In particular, we examine the causal effect of household very low food security status in spring kindergarten on child obesity and overweight status in the spring of fifth grade. The analysis contains two stages. In the first stage, we assess the nature of selection into food insecurity status. In the second stage, we use the nonparametric partial identification method proposed in Kreider et al. (2012) to account for both non-random selection and measurement error in food security status in a single unifying framework. We then turn to a younger sample of children obtained from the Early Childhood Longitudinal Study - Birth Cohort (ECLS-B). Here, we examine the causal effect of household very low food security status at nine months of age on child obesity and overweight status at approximately age five.

The nonparametric partial identification method of Kreider et al. (2012) provides sharp bounds on the average treatment effect (ATE) of very low food security when food security is non-random and potentially measured with error. These bounds require weaker assumptions than those of an instrumental variable (IV), classical measurement error, or linear response model while addressing both selection and measurement error in food security status. However, as a consequence of imposing less structure, we obtain bounds rather than point estimates. Nonetheless, the bounds reveal exactly what can be learned under different assumptions concerning the nature of the selection process and the extent of misreporting. Tamer (2010, p. 168) summarizes the advantages of this approach: "This partial identification approach favors the principle that inference - and conclusions and actions - based on empirical models with fewer suspect assumptions is more robust, hence more sensible and believable. Stronger assumptions will lead to more information about a parameter, but less credible inferences can be conducted."

In terms of the selection problem, we start with the assumption of exogenous selection. We then discuss what can be learned without making any assumptions concerning the selection mechanism; this is the socalled worst-case bounds (Manski 1995). Finally, we impose several monotonicity assumptions: a monotone instrumental variable (MIV) assumption that the latent probability of child obesity and overweight status are nonincreasing in socioeconomic status (SES); a monotone treatment selection (MTS) assumption that children from food insecure households have a higher probability of being obese or overweight compared to food secure children; and a monotone treatment response (MTR) assumption that food insecurity cannot reduce long-run child obesity or overweight status. The MIV assumption is weaker than that required for a typical IV (since the MIV is allowed to have a direct impact on the outcome of interest and may be non-random itself). The MTS assumption posits negative selection into food insecurity, a well established finding in the literature. 
In terms of the measurement error problem, the existing literature on food insecurity states that people may either under-report food insecurity due to social stigma (e.g., Hamelin et al. 2002) or over-report food insecurity if they fear losing access to food stamps or other nutrition assistance (Gundersen and Kreider 2008). We start with the assumption of arbitrary patterns of measurement error ranging from zero to $10 \%$ of the sample. However, since the empirical literature on SNAP and the Special Supplemental Nutrition Program for Women, Infants, and Children (WIC) suggests that eligible participants rarely falsely claim such receipt, we further restrict the assumption on measurement error by imposing the assumption of no false positive errors.

The results are striking and ought to serve as a note of caution and guide to future evaluations of the long-run effects of very low food security on child outcomes. First, in both the ECLS-K and ECLS-B we obtain positive associations between food insecurity and long-run obesity and overweight status when failing to account for non-random selection or misclassification. However, we find strong evidence of nonrandom selection on observed attributes, suggesting that non-random selection on unobserved attributes is likely as well. Unobserved attributes such as household nutritional knowledge, financial management skills, pre- and post-natal health and nutrition, etc. are likely to be associated with both food security and subsequent child health. Second, if even one percent of households misreport their food security status, then the association between food security and future obesity or overweight status cannot be signed even under exogenous selection. Thus, accounting for measurement error is crucial.

Third, bounds that account for selection only - ignoring the possibility of measurement error - exclude zero, indicating a long-run, negative causal effect of very low food security on child obesity when using the ECLS-K data. The ATE cannot be signed when using overweight status as the outcome, or either outcome using the ECLS-B. However, if we redefine the control to include only being food secure (as opposed to defining the control as being not very low food secure), we are able to exclude zero when assessing obesity in the ECLS-K and overweight status in both the ECLS-K and ECLS-B. This negative causal effect indicates that there potentially exists some tension when simultaneously addressing the twin public health issues of food security and child obesity; achieving the former may contribute to the latter.

Finally, bounds accounting for both measurement error and non-random selection fail to sign the ATE for any outcome in either data set without imposing additional assumptions beyond those considered here. We are unable to conclude there exists a long-run causal effect (positive or negative) of very low food security on child weight outcomes even if as few as one percent of households misreport their food security status. These results highlight the importance of accounting for both non-random selection and measurement error in food security status in order to identify the long-run causal impact of very low food security on child health or other outcomes. 
The rest of the paper is organized as follows. Section 2 summarizes the existing literature. Section 3 describes the data. Section 4 describes the methodology. Section 5 discusses the results and offers directions for future research. Section 6 concludes.

\section{Literature Review}

\subsection{Food Security and Child Health}

As noted in the prior section, a growing number of studies have assessed the contemporaneous association between food insecurity and child obesity. Only a handful of studies investigate the long-run consequences of food insecurity. This research also predominantly focuses on associations. Metallinos-Katsaras et al. (2012) assess the association between child weight status at two to five years old and household food insecurity measured at infancy for a sample of low income children participating in the Massachusetts WIC between 2001 and 2006. The authors report that persistent low food insecurity is associated with a $22 \%$ increase in the odds of child obesity compared to those who are persistently food secure. Focusing on a much younger sample of children, Bronte-Tinkew et al. (2007) use data from the 9-month and 24-month waves of the ECLS-B to conclude experiencing very low food security at nine months of age is associated with a higher probability of being overweight at two years of age. The authors also note that very low food security is strongly correlated with infant feeding practices, depressive symptoms, and parenting practices which may explain the higher likelihood of being overweight as a toddler.

Dubois et al. (2006) use data from the Longitudinal Study of Child Development in Québec over the period 1998-2002, reporting that family food insufficiency during preschool years is a strong predictor of obesity and overweight status at 4.5 years of age. Moreover, family food insufficiency during preschool years remains a strong predictor of overweight status at 4.5 years of age even if the child was born with a low birthweight.

Several studies utilize the ECLS-K. Jyoti et al. (2005) assess the effect of food insecurity in both kindergarten and third grade on various child development indicators, including BMI, at third grade. They categorize children based on different combinations of food security and insecurity at the two points in time. The authors find a statistically significant, positive association between kindergarten food insecurity and weight gain for girls, regardless of food security status in the third grade. Bhargava et al. (2008) use the ECLS-K to estimate a dynamic random effects model. The authors conclude that household food insecurity is unlikely to exacerbate child obesity. Finally, Rose and Bodor (2006) assess the relationship between food insecurity in kindergarten and overweight status in first grade and weight gain from kindergarten to first grade. The authors conclude that food insecurity is negatively associated with weight gain, but not 
overweight status. However, their conclusions vary with the definition of food insecurity.

Of the studies assessing the contemporaneous relationship between food insecurity on child BMI, Gundersen and Kreider (2009) merits detailed discussion given the similarity with our analysis. The authors assess the causal effect of food security on child health outcomes while accounting for both non-random selection and measurement error in food security status using pooled cross-sectional data from the 2001-2006 National Health and Nutrition Examination Survey (NHANES) and employing a similar nonparametric bounds approach. The authors obtain bounds that exclude zero under certain monotonicity assumptions and assumptions concerning the misclassification process. Specifically, they find some evidence of a beneficial causal effect of food security on overweight status.

While our study is similar to Gundersen and Kreider (2009), there are important differences. First, we focus on long-run outcomes (i.e., outcomes several years after the measurement of food security). Second, while the age range of children included in their sample is not provided, we examine two specific periods of child development. In particular, the ECLS-B allows us to examine the crucial early post-natal period, while the ECLS-K allows us to examine the crucial period spanning the transition into adolescence (Dietz 1997). Third, although the non-parametric methodology is identical across the two studies and both consider the assumption of arbitrary measurement errors in food security status, we also consider the additional assumption of no false positives in the reporting of food insecurity. Finally, the treatment of primary focus in their study is being marginally food secure or food secure; the control includes being low or very low food secure. In contrast, our baseline analysis defines the treatment as very low food secure and thus the control includes marginally food secure, low food secure, and food secure. We consider their split between the treatment and control in our supplemental analyses.

\subsection{Non-Random Selection and Measurement Error}

None of the above longitudinal studies provide evidence on the long-run causal effect of food insecurity and child health since they fail to simultaneously address the selection and measurement error issues. In terms of selection, Coleman et al. (2012) report that food insecurity rates were substantially higher than the national average for poor households with children of single parents, black and Hispanic households, and households in large cities and rural areas. Thus, food insecure children are not randomly selected. These characteristics, in turn, are correlated with worse health outcomes such as greater incidence of obesity (e.g., Forshee et al. 2004).

However, food insecurity and poverty are not synonymous. For instance, Gundersen et al. (2011) document that a significant number of poor households are food secure; almost $65 \%$ of households around the poverty line are food secure. Moreover, a substantial number of non-poor households are food insecure; 
households with an income-to-poverty ratio close to two have a food insecurity rate above $20 \%$, and households with an income-to-poverty ratio of around three have food insecurity rates of close to $10 \%$. As such, it is quite likely that unobserved attributes such as nutrition and health knowledge, financial literacy, social networks, etc. may be associated with both food insecurity and obesity and overweight status in adults and children. Thus, selection into food insecurity depends on more than observed socioeconomic attributes.

Turning to measurement error, Bound et al. (2001) summarize the causes and consequences of measurement error, concluding that response error, while rampant across a wide range of topics, do not tend to occur randomly. Rather, response errors are quite often correlated with the variable of interest and other common socioeconomic characteristics. In the current context, there are several reasons to question the reliability of the self-reported food insecurity status in household surveys. First, a substantial amount of measurement error has been documented in areas similar to food security such as SNAP participation (Kreider et al. 2012), WIC participation (Kreider et al. 2012; Roy 2012), NSLP participation (Gundersen et al. 2012), disability status and employment (Kreider and Pepper 2007, 2008), health insurance (Kreider and Hill 2009), and child care subsidy receipt (Johnston and Herbst 2013).

Second, evidence specifically regarding measurement error in food insecurity status abounds. For example, in a sample of SNAP recipients only, Gundersen and Kreider (2008) find that recipients may misreport being food insecure if they fear that reporting otherwise might jeopardize their eligibility. On the other hand, Hamelin et al. (2002) hypothesize that parents may misreport being food secure due to embarrassment. Aside from the direction of the mismeasurement, Gundersen and Kreider (2008) take advantage of the sequential nature of the questions in the food security module to look for inconsistencies across responses. They conclude that over $6 \%$ of the sample exhibits at least one inconsistency. Gundersen and Ribar (2011) also note that measurement error can stem from subjective differences in how questions in the CFSM are perceived.

\section{Data}

Data come from the ECLS-K and ECLS-B, collected by the National Center for Education Statistics. The ECLS-K surveys a nationally representative cohort of children throughout the U.S. in fall and spring kindergarten, fall and spring first grade, spring third grade, spring fifth grade, and spring eighth grade. The sample includes data on over 20,000 students who entered kindergarten in one of roughly 1,000 schools during the 1998-99 school year. We retain children for whom we have valid measures of age, gender, 
height, and weight in fifth grade. ${ }^{3}$ The ECLS-B collects information on a nationally representative cohort of roughly 10,700 children born in 2001 at nine months of age, two years, four years, and five years. Both surveys collect detailed family background information, as well as height, weight, and food security. We retain children for whom we have valid measures of age, gender, height, and weight during the final wave. In addition, in both cases we limit the samples by excluding households in highest quintile of SES. ${ }^{4}$

From the information on height and weight of the children, we create BMI $z$-scores. We convert $z$-scores to percentiles. Note that $z$-scores and percentiles are based on CDC 2000 growth charts; these are ageand gender-specific, are adjusted for normal growth, and percentiles are based on the underlying reference population. ${ }^{5}$ Obesity (overweight) is defined as being above the $95^{\text {th }}\left(85^{\text {th }}\right)$ percentile.

The official food security rate is defined over the preceding 12 months. In both surveys, it is calculated on the basis of households' responses to a list of 18 questions in the CFSM for families with children. ${ }^{6}$ The CFSM is a survey module used by the USDA (Nord et al. 2009). The questions aim to capture certain aspects of food insecurity and vary in terms of the severity of the outcome. For example, "We worried whether our food would run out before we got money to buy more" is the least severe outcome while "Did you or other adults in the household ever cut the size of your meals or skip meals because there wasn't enough money for food?" is more severe. The most severe food insecurity outcome captured in the CFSM is: "Did any of the children ever not eat for a whole day because there wasn't enough money for food?" Some of the questions inquire about the frequency with which a certain aspect of food insecurity manifests itself. It is important to note that each of these questions assumes that the condition is due to financial constraints. Table A1 in the appendix presents the CFSM.

The earliest wave of the ECLS-K containing responses to the CFSM is spring kindergarten. The ECLSB contains responses to the CFSM beginning in the initial wave. Utilizing this information, we obtain three measures of food insecurity following official definitions. First, a household with children is classified as

\footnotetext{
${ }^{3}$ We do not examine eighth grade outcomes due to the high attrition rate of children during the transition to middle school for many children.

${ }^{4}$ The initial sample size of the ECLS-K is 21,260. After cleaning age, weight, and height as described in Millimet and Tchernis (2013, Appendix C), and due to sample attrition, the sample size falls to 9,360 in the fifth grade wave. Restricting the sample to a balanced panel reduces the sample size to approximately 9,160. Excluding children from households in the top quintile of SES reduces the sample to 6,470. All samples are rounded to the nearest 10 per NCES restricted data guidelines. In the ECLS-B the possible sample size is roughly 6,950; the initial sample size in the first wave is about 10,700. Restricting the sample to those with valid data on age, gender, height, and weight reduces the sample size to approximately 5,450 . Excluding children from households in the top quintile of SES reduces the sample to 4,100. Note, all sample sizes are rounded to the nearest 50 per NCES restricted data regulations for the ECLS-B.

${ }^{5} z$-scores and their percentiles are obtained using the -zanthro- command in Stata.

${ }^{6}$ Families without children and one-member households face a subset of ten questions.
} 
marginally food secure if it affirms at least one question in the CFSM. Second, a household with children is classified as low food secure if it affirms at least three questions in the CFSM. Finally, a household with children is classified as very low food secure if it affirms eight or more questions in the CFSM. We focus primarily on very low food security as this obviously represents the most dire situation. In addition, this category has witnessed relatively greater growth of late with the prevalence rate among households increasing from $3.7 \%$ to $5.7 \%$ from 1998 to 2011; the corresponding change for low food secure households is from $8.1 \%$ to $9.2 \%$ (Coleman-Jensen et al. 2012). Put differently, the number of very low food secure households increased by $78 \%$ over this period, whereas the corresponding increase for low food secure households was $32 \%$.

Tables A2 and A3 in the appendix provide summary statistics. In the ECLS-K, $19.2 \%$ report being marginally food secure, $9.9 \%$ report being low food secure, and $1.9 \%$ report being very low food secure. $43.2 \%$ of children are overweight in the spring of fifth grade; $24.2 \%$ of children are obese. In the ECLS-B, the corresponding figures for food security status are $13.3 \%, 12.8 \%$, and $3.4 \%$. Furthermore, $34.6 \%$ of children are overweight at roughly age five; $17.1 \%$ of children are obese. The observed characteristics in both samples illustrate that food insecure children, relative to food secure children, are more likely to belong to single parent households, have more siblings, and have a less educated mother. Since SNAP aims to fight hunger by alleviating food insecurity, it is not surprising that food insecure children are more likely to belong to households participating in SNAP. The observed characteristics thus indicate strong adverse selection into food insecurity. It is possible that salient unobserved factors such as inadequate nutrition knowledge, unhealthy food habits, and poor financial management skills also increase the likelihood of bad health as well as being food insecure.

\section{Methodology}

Our objective is to bound the ATE of being food insecure on future weight status. The ATE captures the expected effect of food insecurity (relative to not food insecure) for a random child chosen from the underlying population. ${ }^{7}$ With binary outcomes, the ATE is defined as

$$
\operatorname{ATE}(1,0)=P\left[H\left(F I^{*}=1\right)=1 \mid X \in \Omega\right]-P\left[H\left(F I^{*}=0\right)=1 \mid X \in \Omega\right]
$$

\footnotetext{
${ }^{7}$ In this section, child's weight status implicitly refers to our long-run measures of obesity and overweight status. Food insecurity refers to a measure of household food insecurity (i.e., marginally food secure, low food secure, or very low food secure) obtained in the initial period (spring kindergarten in the ECLS-K and 9-months of age in the ECLS-B). For ease of exposition, we will refer to the child being food insecure (or not) although food security is determined at the household level.
} 
where $P[\cdot]$ denotes the probability of the argument being true, $H$ is a binary indicator defined such that one (zero) denotes a bad (good) health outcome (e.g., obese or overweight), and $F I^{*}$ is a binary indicator defined such that one (zero) corresponds to actual food insecurity (security). The probabilities are conditioned on observed covariates denoted by $X \in \Omega$ with values in the set $\Omega$. In this approach, conditioning on covariates only helps to define subpopulations of interest (Kreider et al. 2012). For notational simplicity, $X \in \Omega$ is dropped in the following derivations. Furthermore, from here on let $H(1) \equiv H\left(F I^{*}=1\right)$ and $H(0) \equiv H\left(F I^{*}=0\right)$, where $H(1)$ and $H(0)$ represent potential outcomes.

To assess the causal effect of food insecurity on a child's health using observational data, two identification problems must be addressed. The first is the well-known problem of the missing counterfactual. For instance, we do not observe the probability of an adverse health outcome for food insecure children if instead they had been food secure. This is referred to as the selection problem. To see this, note that by the Law of Total Probability we can write

$$
\begin{aligned}
P[H(1)=1]= & P\left[H(1)=1 \mid F I^{*}=1\right] P\left(F I^{*}=1\right)+ \\
& P\left[H(1)=1 \mid F I^{*}=0\right] P\left(F I^{*}=0\right) .
\end{aligned}
$$

If actual food insecurity status is observed, the sampling process identifies $P\left(F I^{*}=1\right)$ and $P\left(F I^{*}=0\right)$ and the expected outcome conditional on the outcome being observed, $P\left[H(1)=1 \mid F I^{*}=1\right]$. However, the sampling process fails to identify the average outcome for those not food insecure, $P\left[H(1)=1 \mid F I^{*}=0\right]$. Thus, $P[H(1)=1]$ is not nonparametrically identified. A similar result holds for $P[H(0)=1]$.

The second identification problem arises if actual food insecurity status is not observed for all respondents. Let FI denote the observed, self-reported indicator of food insecurity status, where FI equals one if the household reports being food insecure and zero otherwise. This is referred to as the measurement or misclassification error problem. With misclassification the sampling process fails to provide any useful information on actual food insecurity status, $F I^{*}$, absent assumptions on the extent and type of measurement error. In this case, all quantities on the right hand side of equation (2) are unknown.

Let the latent variable $Z^{*}$ denote whether a report is accurate or not; $Z^{*}$ equals one if $F I^{*}=F I$ and zero otherwise. Kreider et al. (2012) show that $P[H(1)=1]$ may be decomposed as follows:

$$
\begin{aligned}
P[H(1)=1]= & {\left[P(H=1, F I=1)-\theta_{1}^{+}+\theta_{1}^{-}\right]+} \\
& P\left[H=1 \mid F I^{*}=0\right]\left[P(F I=0)+\left(\theta_{1}^{+}+\theta_{0}^{+}\right)-\left(\theta_{1}^{-}+\theta_{0}^{-}\right)\right]
\end{aligned}
$$

where $H$ is the observed (realized) health outcome, $\theta_{j}^{+} \equiv P\left(H=j, F I=1, Z^{*}=0\right)$ and $\theta_{j}^{-} \equiv P(H=$ $\left.j, F I=0, Z^{*}=0\right)$ represent the proportion of false positive and false negative classifications of food 
insecure children, respectively, for children realizing health outcome $j=1,0 .{ }^{8}$ Examination of (3) reveals that all of the terms on the right hand side except $P(H=1, F I=1)$ and $P(F I=0)$ are unobserved. $P\left[H=1 \mid F I^{*}=0\right]$ is not identified since $F I^{*}$ is unobserved and the $\theta$ terms are not identified since $Z^{*}$ is unobserved.

Given the lack of nonparametric identification of the ATE, bounds are derived by combining various assumptions concerning the nature of the selection process along with two assumptions about the nature and extent of measurement error.

\subsection{Classification Error Assumptions}

When considering measurement error, we allow for two cases. In the first case, we place no structure on the pattern of reporting errors. We refer to this case as arbitrary errors. In the second case, we impose some structure by assuming that no households falsely report being food insecure. We refer to this as the case of no false positives. Due to social stigma or embarrassment, many households may overstate their level of food security (Hamelin et al. 2002). However, it is also possible that some households falsely report being food insecure if they believe that not doing so may hurt their eligibility for food assistance programs such as SNAP (Gundersen and Kreider 2009). That said, since individuals tend to underreport SNAP and WIC participation in household surveys, the assumption of no false positive errors appears to be a reasonable restriction (e.g., Kreider et al. 2012; Roy 2012).

Formally, we follow Gundersen and Kreider (2008) and impose the following assumptions pertaining to classification errors:

(A1) Upper Bound Error Rate Assumption: $P\left(Z^{*}=0\right) \leq Q$

(A2) No False Positives Assumption: If $F I=1$, then $Z^{*}=F I^{*}=1$.

Here, $Q$ is an upper bound on the degree of misclassification. It takes a value of zero if one wishes to rule out the possibility of measurement error in self-reported food security status. The second assumption states that self-reports of food insecurity are presumed to be accurate; no such assumption is made concerning self-reports of food security. The arbitrary errors case imposes only Assumption A1; the no false positives case imposes both Assumptions A1 and A2. In both cases, we vary values of $Q$, setting $Q=0,0.01,0.02$, 0.05 , and 0.10 .

The above assumptions on classification errors impose reasonable restrictions on the unknown misclas-

\footnotetext{
${ }^{8}$ The formulae and their derivations come from an earlier version of Kreider et al. (2012).
} 
sification rates $\theta_{1}^{-}, \theta_{0}^{-}, \theta_{1}^{+}$, and $\theta_{0}^{+}$. Assumption $\mathrm{A} 1$ implies

$$
\begin{aligned}
& 0 \leq \theta_{0}^{-} \leq \min \{Q, P(H=0, F I=0)\} \equiv \theta_{0}^{U B-} \\
& 0 \leq \theta_{1}^{-} \leq \min \{Q, P(H=1, F I=0)\} \equiv \theta_{1}^{U B-} \\
& 0 \leq \theta_{0}^{+} \leq \min \{Q, P(H=0, F I=1)\} \equiv \theta_{0}^{U B+} \\
& 0 \leq \theta_{1}^{+} \leq \min \{Q, P(H=1, F I=1)\} \equiv \theta_{1}^{U B+}
\end{aligned}
$$

and

$$
\theta_{1}^{+}+\theta_{1}^{-}+\theta_{0}^{+}+\theta_{0}^{-} \leq Q
$$

Assumption A2 implies

$$
\theta_{1}^{+}=\theta_{0}^{+}=0
$$

\subsection{Exogenous Selection}

\subsubsection{No Misclassification Errors}

Since the existing literature on the long-run consequences of food insecurity typically assumes exogenous selection into food insecurity, this assumption provides a usual starting point for the analysis. The assumption of exogenous selection is expressed as

$$
P\left[H(1)=1, F I^{*}\right]=P[H(1)=1]
$$

which implies

$$
P\left[H(1)=1, F I^{*}=1\right]=P\left[H(1)=1, F I^{*}=0\right]=P[H(1)=1] .
$$

Accordingly, using (2) implies

$$
\begin{aligned}
& P[H(1)=1]=P\left[H=1 \mid F I^{*}=1\right] \\
& P[H(0)=1]=P\left[H=1 \mid F I^{*}=0\right] .
\end{aligned}
$$

Finally, with exogenous selection and no misclassification errors, the ATE is nonparametrically identified and given by

$$
\begin{aligned}
A T E & =P[H(1)=1]-P[H(0)=1] \\
& =P\left[H=1 \mid F I^{*}=1\right]-P\left[H=1 \mid F I^{*}=0\right] .
\end{aligned}
$$




\subsubsection{Misclassification Errors}

Allowing for misclassification, the $A T E$ is no longer nonparametrically identified even under the assumption of exogenous selection as $F I^{*}$ is not observed in (6). To illustrate, note that

$$
P[H(1)=1]=P\left[H=1 \mid F I^{*}=1\right]
$$

can be decomposed as

$$
P[H(1)=1]=\frac{P(H=1, F I=1)+\theta_{1}^{-}-\theta_{1}^{+}}{P(F I=1)+\left(\theta_{1}^{-}+\theta_{0}^{-}\right)-\left(\theta_{1}^{+}+\theta_{0}^{+}\right)}
$$

where the sampling process identifies only $P(H=1, F I=1)$ and $P(F I=1)$. The term $\left(\theta_{1}^{-}+\theta_{0}^{-}\right)-$ $\left(\theta_{1}^{+}+\theta_{0}^{+}\right)$, in the denominator, denotes the unobserved excess of false negatives over false positives in the population. The term $\left(\theta_{1}^{-}-\theta_{1}^{+}\right)$, in the numerator, reflects the excess of false negatives over false positives among those children with $H=1$.

To derive bounds on the ATE under different assumptions concerning the nature and extent of the misclassification errors, recall that ATE is given by

$$
A T E=P[H(1)=1]-P[H(0)=1]
$$

Thus, the bounds for the ATE are given by

$$
\begin{aligned}
U B_{A T E} & =U B_{P[H(1)=1]}-L B_{P[H(0)=1]} \\
L B_{A T E} & =L B_{P[H(1)=1]}-U B_{P[H(0)=1]}
\end{aligned}
$$

where $U B$ and $L B$ denote the upper and lower bounds, respectively.

With arbitrary errors, Kreider and Pepper (2007) derive the following expressions for the bounds:

$$
\begin{aligned}
U B_{A T E} & =\sup _{a \in(0, \min [Q, P(H=1, F I=0)])}\left[\frac{P[H=1, F I=1]+a}{P(F I=1)+2 a-Q}-\frac{P[H=1, F I=0]-a}{P(F I=0)-2 a+Q}\right] \\
L B_{A T E} & =\inf _{b \in(0, \min [Q, P(H=1, F I=1)])}\left[\frac{P[H=1, F I=1]-b}{P(F I=1)-2 b+Q}-\frac{P[H=1, F I=0]+b}{P(F I=0)+2 b-Q}\right] .
\end{aligned}
$$

Under the assumption of no false positives, the individual components of the ATE, given in (8), are bounded as follows

$$
\begin{array}{r}
\frac{P[H=1, F I=1]}{P(F I=1)+\theta_{0}^{U B-}} \leq P[H(1)=1] \leq \frac{P[H=1, F I=1]+\theta_{1}^{U B-}}{P(F I=1)+\theta_{1}^{U B-}} \\
\frac{P[H=1, F I=0]-\theta_{1}^{U B-}}{P(F I=0)-\theta_{1}^{U B-}} \leq P[H(0)=1] \leq \frac{P[H=1, F I=0]}{P(F I=0)-\theta_{0}^{U B-}} .
\end{array}
$$

Accordingly, the bounds on the ATE are given by

$$
\begin{aligned}
U B_{A T E} & =\frac{P[H=1, F I=1]+\theta_{1}^{U B-}}{P(F I=1)+\theta_{1}^{U B-}}-\frac{P[H=1, F I=0]-\theta_{1}^{U B-}}{P(F I=0)-\theta_{1}^{U B-}} \\
L B_{A T E} & =\frac{P[H=1, F I=1]}{P(F I=1)+\theta_{0}^{U B-}}-\frac{P[H=1, F I=0]}{P(F I=0)-\theta_{0}^{U B-}} .
\end{aligned}
$$




\subsection{No Selection Assumption}

The bounds given in (11) - (14) invoke the assumption of exogenous selection which is highly improbable. Consequently, we next consider what can be learned about the ATE of food insecurity on child health without invoking any assumptions concerning selection into the treatment following Manski (1995).

\subsubsection{No Misclassification Errors}

In the absence of measurement error, but with no assumptions concerning selection, the only information available concerning the missing counterfactuals are that they lie in the unit interval since they represent probabilities; formally, $P\left[H(1)=1 \mid F I^{*}=0\right], P\left[H(0)=1 \mid F I^{*}=1\right] \in[0,1]$. Accordingly, the individual components of the ATE are bounded as follows

$$
\begin{aligned}
& P\left[H=1, F I^{*}=1\right] \leq P[H(1)=1] \leq P\left[H=1, F I^{*}=1\right]+P\left(F I^{*}=0\right) \\
& P\left[H=1, F I^{*}=0\right] \leq P[H(0)=1] \leq P\left(F I^{*}=1\right)+P\left[H=1, F I^{*}=0\right] .
\end{aligned}
$$

Note, the width of the bounds on $P[H(1)=1]$ is the censoring probability, $P\left(F I^{*}=0\right)$, while the width of the bounds on $P[H(0)=1]$ is the inclusion probability, $P\left(F I^{*}=1\right)$. As a result, although the bounds on ATE are sharp, the width always equals unity and includes zero (Manski 1995). So, without identifying restrictions on the selection mechanism, it is impossible to sign the ATE. While the sign is unknown, extreme values are excluded from the bounds, thus providing some potentially useful information.

\subsubsection{Misclassification Errors}

Allowing for measurement error, the bounds on individual components of the ATE become

$$
\begin{aligned}
& P[H=1, F I=1]-\theta_{1}^{+}+\theta_{1}^{-} \leq P[H(1)=1] \leq P[H=1, F I=1]+P(F I=0)+\theta_{0}^{+}-\theta_{0}^{-} \\
& P[H=1, F I=0]+\theta_{1}^{+}-\theta_{1}^{-} \leq P[H(0)=1] \leq P[H=1, F I=0]+P(F I=1)-\theta_{0}^{+}+\theta_{0}^{-} .
\end{aligned}
$$

With arbitrary errors, the bounds on ATE are given by

$$
\begin{aligned}
U B_{A T E} & =P[H=1, F I=1]+P(F I=0)+\min \left\{Q, \theta_{0}^{U B+}+\theta_{1}^{U B-}\right\}-P[H=1, F I=0] \\
L B_{A T E} & =P[H=1, F I=1]-\min \left\{Q, \theta_{1}^{U B+}+\theta_{0}^{U B-}\right\}-P[H=1, F I=0]-P(F I=1) .
\end{aligned}
$$

Under the assumption of no false positives, the bounds become potentially tighter and are given by

$$
\begin{aligned}
U B_{A T E} & =P[H=1, F I=1]+P(F I=0)+\theta_{1}^{U B-}-P[H=1, F I=0] \\
L B_{A T E} & =P[H=1, F I=1]-\theta_{0}^{U B-}-P[H=1, F I=0]-P(F I=1) .
\end{aligned}
$$




\subsection{Monotonicity Assumptions}

While the bounds given in (15) - (18) have the advantage of not invoking any assumptions concerning the selection process into actual treatment assignment, they have the disadvantage of never being able to exclude zero from the bounds. Thus, the sign of the ATE cannot be learned. To tighten the bounds on ATE, without going so far as to assume exogenous selection, we assess the identifying power of three monotonicity assumptions which impose different restrictions on the relationships between food insecurity, child health outcomes, and the available data.

\subsubsection{Monotone Treatment Selection}

The Monotone Treatment Selection (MTS) assumption places some structure on the relationship between potential outcomes and treatment assignment (Manski and Pepper 2000). Specifically, the MTS assumption posits that children from food insecure households have worse potential outcomes on average compared to children from food secure households. This assumption is relatively innocuous given the vast literature documenting less favorable demographic, socioeconomic, and health characteristics in food insecure households (e.g., Gundersen and Kreider 2009). Following Kreider et al. (2012), this assumption translates to

$$
\begin{aligned}
& P\left[H(1)=1 \mid F I^{*}=1\right] \geq P\left[H(1)=1 \mid F I^{*}=0\right] \\
& P\left[H(0)=1 \mid F I^{*}=1\right] \geq P\left[H(0)=1 \mid F I^{*}=0\right]
\end{aligned}
$$

since $H(\cdot)=1$ represents a worse health outcome (i.e., obese or overweight). Imposing MTS, the bounds on ATE are given by

$$
\begin{aligned}
U B_{A T E} & =\frac{P[H=1, F I=1]+\theta_{1}^{U B-}}{P(F I=1)+\theta_{1}^{U B-}-\theta_{0}^{U B+}}-\frac{P[H=1, F I=0]-\theta_{1}^{U B-}}{P(F I=0)+\theta_{0}^{U B+}-\theta_{1}^{U B-}} \\
L B_{A T E} & =P[H=1, F I=1]-\theta_{1}^{U B+}-\left\{P[H=1, F I=0]+P(F I=1)+\theta_{0}^{U B-}\right\}
\end{aligned}
$$

where $\theta_{1}^{U B+}=\theta_{0}^{U B-}=\theta_{1}^{U B-}=\theta_{0}^{U B+}=0$ in the absence of measurement error.

With arbitrary errors, the upper bound of the ATE is given by the upper bound of the ATE under exogenous selection with arbitrary errors in equation (11). The lower bound is given by the lower bound under no selection assumptions with arbitrary errors in equation (16). Under the assumption of no false positives, the bounds are obtained from the same models, however using the corresponding no false positive assumption (i.e., Equations (13) and (18)). 


\subsubsection{Monotone Instrumental Variable}

To further tighten the bounds, we turn to the Monotone Instrumental Variable (MIV) assumption which makes use of new information through the introduction of a monotone instrumental variable. A MIV should not be confused with a typical instrumental variable. The only requirement for a MIV is that potential outcomes vary monotonically with the variable (Manski and Pepper 2000). Following Kreider et al. (2012), the MIV assumption imposes

$$
\begin{aligned}
& P\left[H(1)=1 \mid \nu=u_{2}\right] \leq P[H(1)=1 \mid \nu=u] \leq P\left[H(1)=1 \mid \nu=u_{1}\right] \\
& P\left[H(0)=1 \mid \nu=u_{2}\right] \leq P[H(0)=1 \mid \nu=u] \leq P\left[H(0)=1 \mid \nu=u_{1}\right]
\end{aligned}
$$

where $v$ is the MIV and $u_{1}<u<u_{2}$. In other words, lower values of $v$ are associated with worse potential outcomes (again, since $H(\cdot)=1$ represents a worse health outcome). Here, we use household SES as the MIV. A lengthy literature documents the positive income-health gradient for children (e.g., Case et al. 2002).

To proceed, we combine the MIV and MTS (with and without measurement error) assumptions. Let $U B(u)$ and $L B(u)$ denote the upper and lower bounds of the individual components of the ATE obtained under a set of MTS and measurement error assumptions evaluated conditional on $v=u$. As a result, the joint MTS-MIV assumption implies

$$
\sup _{u_{2} \geq u} L B\left(u_{2}\right) \leq P[H(t)=1 \mid \nu=u] \leq \inf _{u_{1} \leq u} U B\left(u_{1}\right), t=0,1 .
$$

See Proposition 1 in Manski and Pepper (2000).

To calculate these bounds in practice, the sample is divided into four SES cells. Weighted averages of the estimates of the $U B$ and $L B$ across the four cells yield joint MTS-MIV bounds on the individual components of the ATE. Final bounds for the ATE are then computed using (9) and (10). The MIV estimator is biased in finite samples, but consistent (Manski and Pepper 2000). In light of this, we use Kreider and Pepper's (2007) nonparametric finite sample bias-corrected MIV estimator.

\subsubsection{Monotone Treatment Response}

The final monotonicity assumption is the Monotone Treatment Response (MTR) assumption. This assumption relates to the expected relationship between the treatment and outcome (Manski 1997). Specifically, the MTR assumption posits that food insecurity cannot improve health outcomes. Formally, this implies that $H(1) \geq H(0)$; i.e., the probability of a bad health outcome must be at least as high under food 
insecurity as food security. To be clear, the MTS assumption states that food insecure children are comparatively more disadvantaged on average than their food secure counterparts, so they have worse outcomes on average independent of food insecurity. The MTR assumption, on the other hand, states that becoming food insecure would not improve a child's weight status. While we assess the information content of this assumption, it is not obvious that the assumption is true. While one would expect food insecurity to adversely impact long-run general health, the impact on weight status is unclear due to opposing forces (Gundersen and Kreider 2009). On the one hand, food insecurity limits food intake which should reduce child weight, at least in the short-run. On the other hand, food insecurity may alter the nutritional content of the food consumed, encourage overeating when food is available, and contribute to metabolic changes in children, thereby contributing to weight gain in the long-run. That said, the practical implication of the MTR assumption in the current context is that it tightens the bounds on the ATE by excluding all negative values since equation (8) must be non-negative.

\section{Results}

\subsection{Baseline}

The baseline set of empirical results are presented in Figures 1-4 and Table 1-4. In all cases, the treatment is very low food security and the control group includes all other children (i.e., children from food secure, marginally food secure, and low food secure households). Figures 1 and 2 and the associated tables utilize data from the ECLS-K; Figures 3 and 4 and the associated tables utilize data from the ECLS-B. Figures 1 and 3 and the corresponding tables define the outcome as equal to one if the child is obese, zero otherwise; Figures 2 and 4 and the corresponding use overweight status as the outcome. Finally, to address the uncertainty arising from sampling variability, the tables report Imbens-Manski (2004) confidence intervals that cover the true value of the ATE with 95\% probability (see Kreider et al. 2012).

In each figure, the left panel compares the sharp bounds on the ATE obtained under the assumption of exogenous selection to those obtained under no assumption on selection as $Q$ varies from 0 to 0.10 . The middle (right) panel reports the bounds under different combinations of the monotonicity assumptions concerning selection under the arbitrary errors (no false positives) assumption concerning measurement error. The corresponding tables report the actual values along with the confidence intervals.

Turning to the results, several findings stand out. First, in all four tables, the ATE is positive, but

not statistically significant, under the assumptions of exogenous selection and no measurement error. The lack of statistical significance is not surprising given the small number of children experiencing very low food security. In the ECLS-K, very low food security during kindergarten is associated with a $3.1 \%(4.1 \%)$ 
increase in the probability of a child being obese (overweight) in fifth grade. In the ECLS-B, very low food security at nine months of age is associated with a $6.1 \%(6.6 \%)$ increase in the probability of a child being obese (overweight) at approximately age five. Given the difference in observed characteristics between children in very low food secure households and all other children, this positive association is not surprising.

Second, the impact of misreporting is profound. If even one percent of the sample misreports their food security status, the sign of the ATE cannot be determined even under exogenous selection. Given prior data on misreporting in other contexts (e.g., Bound et al. 2001), combined with the sensitive nature of the food security questionnaire, this is a stark result. Third, without imposing any assumptions concerning the selection process, the bounds are of width one and necessarily include zero as discussed above under the assumption of no measurement error. Nonetheless, the bounds are useful in excluding possible values of the ATE. For instance, Table 1 reveals bounds on the ATE for obesity using the ECLS-K of [-0.251, 0.749]. Table 3 reveals bounds of $[-0.189,0.811]$ using the ECLS-B. Thus, a considerable range of values of the ATE, particularly in the negative domain, are ruled out. These bounds become wider as greater amounts of measurement error are allowed; the corresponding bounds are $[-0.351,0.849]$ and $[-0.289,0.911]$ if $Q=0.10$. However, it is interesting to note that the assumption of no false positives has no identifying power relative to the assumption of arbitrary errors over the range of permissible values of $Q$ utilized.

Fourth, the monotonicity assumptions are quite powerful in terms of tightening the bounds. MTS results in significant shrinkage of the upper bounds; MIV further reduces the upper bounds. MTR raises the lower bounds quite substantially, but in an obvious way (it simply assumes away negative values for the ATE). That said, even in the absence of measurement error, the point estimates include zero in all cases except one. In Table 1, the joint MTS-MIV bounds are [-0.252, -0.018]; the Imbens-Manski (2004) confidence intervals, however, include zero. Nonetheless, this is suggestive of a negative, long-run effect of very low food security on child obesity. This has important policy implications. While certainly no one would advocate inducing food insecurity to combat the obesity epidemic, the results suggest that there exists some tension in simultaneously combating hunger and obesity.

Finally, it is worth noting that even upon invoking the various monotonicity assumptions, even small rates of misreporting significantly widen the bounds. However, in combination with the monotonicity assumptions, the assumption of no false positives has significant identifying power. For example, in Tables 1 and 2, the upper bound under MTS alone attains its maximum possible value of unity when $Q=0.02$ under arbitrary errors; this occurs at $Q=0.05$ in Tables 3 and 4 under arbitrary errors. However, the upper bound, while still high, never exceeds 0.75 under MTS and the assumption of no false positives.

In summary, we find that the ATE of very low food security in kindergarten (relative to not very low 
security) has a negative causal effect on obesity in fifth grade under the minimal assumptions of MTS-MIV only when we assume food security is reported without error. However, it is not possible to sign long-run relationship between very low food security in kindergarten and child overweight status in the fifth grade, nor very low food security at nine months of age on child obesity or overweight status at five years of age, with or without measurement error in self-reported household food security status. Moreover, our results illustrate the difficulty in not only narrowing the range of plausible values for the ATE, but even estimating its sign, in the absence of strong assumptions regarding selection and the lack of measurement error.

\subsection{Additional Analyses}

We undertake several additional analyses to see what can be learned under the set of assumptions considered here when we alter the parameter being bounded. As stated previously, in the baseline analyses we estimate bounds for the ATE of being very low food secure relative to not being very low food secure. Thus, the control consists of any level of food security in the initial period except very low food secure. As a result, being low food secure or marginally food secure, in addition to food secure, comprise the control. Our first supplemental analysis maintains the same treatment group - children in very low food secure households but restricts the control to only food secure children. Our second supplemental analysis alters the treatment group as well as the control group. Here, we first define the treatment as being low or very low food secure, retaining marginally food secure and food secure as the control, and second define the treatment as being marginally, low, or very low food secure, retaining only food secure as the control. Our final supplemental analysis defines the treatment as low or very low food secure, but includes only food secure as the control.

\subsubsection{Alternative Control}

Bounds for the ATE of being very low food secure relative to food secure are presented in Figures 5-8 and Tables 5-8. These figures and tables are analogous to those in Figures 1-4 and Tables 1-4. The only difference is that now the control group excludes children in low and marginally food secure households. Because of the greater disparity between the treatment and control groups in terms of the provision of food, one might expect a more stark causal effect of the treatment. ${ }^{9}$

Turning to the results, many of the findings from the baseline results continue to hold. Thus, in the interest of brevity, we focus on the one main difference. In the baseline case, the MTS-MIV bounds under

\footnotetext{
${ }^{9}$ It is important to remember that the causal effect of a treatment is only defined with respect to a specific control. As the control differs from that used in the baseline analysis, the parameter being estimated is different. In the baseline analysis we are bounding the ATE of being very low food secure relative to being not very low food secure. Here, we are bounding the ATE of being very low food secure relative to being food secure.
} 
the assumption of no measurement error exclude zero - indicating a negative causal effect on average only when using the ECLS-K to assess obesity status. However, when defining the control as food secure, the point estimates for the bounds exclude zero not only in this case (Figure 5 and Table 5), but also when assessing overweight status in the ECLS-K (Figure 6 and Table 6) and ECLS-B (Figure 8 and Table 8). With the caveat in mind that the Imbens-Manski (2004) confidence intervals include zero, this continues to provide a strong indication of a negative, long-run effect of very low food security on child weight status.

\subsubsection{Alternative Treatment}

Our next analyses assess the causal effect of less extreme forms of food insecurity. First, we bound the ATE of being low or very low food secure relative to being marginally food secure or food secure. These results are presented in Panel I of Tables A4-A7 in the Appendix. ${ }^{10}$ Second, we bound the ATE of being marginally, low, or very low food secure relative to being food secure. These results are presented in Panel II of Tables A4-A7.

It continues to be the case that results are very similar to the baseline results. Thus, we focus on only a few salient findings. First, while the associations between the two treatments and weight status under exogenous selection, assuming no measurement error, continue to be positive when using the ECLS-K, the estimate is also statistically significant in Panel II of Table A4. The associations between the two treatments and weight status under exogenous and no measurement error are very close to zero when using the ECLS-B and, in fact, become negative for both treatments when assessing overweight status (Table A7). Thus, while perhaps not statistically different, there is some evidence that the long-run association between low and marginal food security is closer to zero during the years prior to kindergarten than during early primary school.

Second, the MTS-MIV bounds exclude zero in many cases when no misreporting is assumed. Specifically, we obtain bounds for the ATE of low or very low food security (relative to marginally food secure or food secure) on obesity status of $[-0.289,-0.007]$ and $[-0.276,-0.011]$ in the ECLS-K (Panel I, Table A4) and ECLS-B (Panel I, Table A6), respectively. The bounds for this treatment also exclude zero when assessing overweight status in the ECLS-B (Panel I, Table A7). Finally, bounds for the ATE of marginally, low, or very low food security (relative to food secure) on obesity and overweight status also exclude zero when assessing either obesity (Panel II, Table A6) or overweight (Panel II, Table A7) status in the ECLS-B. Finally, when assessing overweight status in the ECLS-B, MTS alone, along with the assumption of no measurement error, is sufficient to exclude zero from the bounds for both treatments (Panels I and II, Table A7).

\footnotetext{
${ }^{10}$ To conserve space, we omit the corresponding figures.
} 
Our final analysis retains the same treatment from Panel I of Tables A4-A7, namely being either low or very low food secure, but now the control is food secure (rather than marginally food secure or food secure). As in the prior section, this introduces a greater wedge between the treatment and control. The results are presented in Table A8 for the ECLS-K and Table A9 for the ECLS-B. For the ECLS-K, the change in definition of the control affects very little. The point estimates obtained under the exogenous selection assumption and no measurement error are very similar, the MTS-MIV bounds assuming no measurement error exclude zero when assessing obesity status, and the impact of misreporting is of similar magnitude. For the ECLS-B, however, some interesting results emerge. First, under the assumption of no measurement error, MTS-MIV is sufficient to exclude zero from the bounds for both obesity and overweight status. Moreover, the result is statistically significant at the $95 \%$ confidence level for overweight status as the Imbens-Manski (2004) bounds exclude zero (Panel II, Table A9). Second, the bounds continue to exclude zero for overweight status even when some measurement error is allowed $(Q=0.01)$ under either arbitrary errors or no false positives. However, now the confidence intervals fail to exclude zero. Finally, MTS alone is sufficient to exclude zero when assessing overweight status assuming no measurement error.

\subsection{Discussion}

Accounting for adverse selection into various degrees of food insecurity and assuming self-reports of food security status are accurate, the results provide strong, albeit often not statistically significant, evidence of a long-run, negative causal effect of food insecurity on child obesity and overweight status. The fact that the point estimates of the bounds are often able to exclude zero highlights what can be learned under minimal monotonicity assumptions. More importantly, the results suggest that the twin public health goals of ameliorating hunger and reducing childhood obesity may be somewhat at odds, at least in the near term. Finally, the results point to the substantial loss of information from relatively little measurement error. Researchers in this area (and others) should heed this warning. It is not sufficient to overlook measurement error under the rationale that it is a relatively 'minor' problem. With even one percent of the sample misreporting their food security status, the width of the bounds can increase markedly.

Returning to the long-run, negative causal effect often suggested by the analysis under the assumption of no measurement error, further discussion is warranted. In particular, it is noteworthy that the study most similar to this by Gundersen and Kreider (2009) obtain bounds providing some evidence that food security reduces the probability of children being overweight. As stated previously, there are several differences between their study and ours. First, the definitions of the treatment, control, and outcome in their primary analysis correspond most closely to Panel I of Table A5 in the appendix. Specifically, 
they bound the ATE of being marginally food secure or food secure relative to being low or very low food secure on contemporaneous overweight status. Second, the age ranges of the samples may differ; it is not reported in their study. Third, and most importantly, the bounds in Gundersen and Kreider (2009) typically exclude zero only when they impose MTR in addition to MTS-MIV. As stated above (and in Gundersen and Kreider (2009)), the validity of the MTR assumption when analyzing weight outcomes is highly questionable. It is noteworthy that when they impose MTS-MIV only, along with the assumption of no measurement error, they obtain bounds for the ATE of low food security on contemporaneous overweight status of $[-0.424,0.039]$, whereas our corresponding bounds for the ATE on long-run overweight status are $[-0.444,0.007]$ and $[-0.397,-0.016]$ in the ECLS-K and ECLS-B, respectively. Moreover, in the ancillary analysis in Gundersen and Kreider (2009), they also estimate bounds for the ATE of very low food security on contemporaneous overweight and obesity status under MTS-MIV alone. Under the assumption of no measurement error, they obtain bounds of $[-0.368,0.015]$ and $[-0.245,0.019]$, respectively. Our corresponding bounds for the ATE on long-run overweight and obesity status are $[-0.434,0.015]$ and $[-0.252,-0.018]$, respectively, in the ECLS-K; $[-0.354,0.029]$ and $[-0.189,0.038]$, respectively, in the ECLS-B. Thus, there is little difference between the short- and long-run results and across the various data sets once the MTR assumption is dropped.

If indeed there is a long-run, negative causal effect of food insecurity on child obesity and overweight status, this would be somewhat surprising. While this is potentially explained by the simple notion that food insecurity results in a reduction in caloric intake, it is perhaps unexpected that this would not be offset by the harmful effects of consuming more energy-dense foods, overeating during times of food availability, or metabolic changes in response to bouts of hunger (Rose and Bodor 2006; Gundersen and Kreider 2009). Future work should investigate the possible mechanisms as it may provide some guidance for public health officials devising policy responses to the obesity epidemic. For example, it may be that food insecure households are more likely to participate in beneficial nutrition assistance programs such as WIC, SNAP, or school meal programs. Or, informal social networks may be relied upon to ensure adequate nutrition during times of need. Finally, it would be beneficial for future work to investigate the causal effect of food insecurity on health outcomes in the even longer run (e.g., into adulthood), as well as assess whether food insecurity during different periods of child development have differential effects. For example, there is increasing evidence that experiences in utero or even prior to conception may shape an individual's health trajectory over their lifetime (Dietz 1997; Martorell et al. 2001). 


\section{Conclusion}

The existing literature on the long-run relationship between food security status and child obesity and overweight status explores only the association between these two major public health concerns instead of the causal relationship. This is because existing studies do not account for two important identification issues: non-random selection and misreporting in household surveys. Here, we revisit the long-run impact of food security on obesity and overweight status, addressing both identification issues in a single partial identification framework proposed in Kreider et al. (2012). This nonparametric approach is especially suitable for this analysis given that obtaining consistent point estimates of an endogenous and mismeasured binary variable is not trivial (Black et al. 2000). Moreover, our study complements prior work on bounding the short-run causal effect of food security on child weight in Gundersen and Kreider (2009).

In the presence of both misreporting of and adverse selection into food insecurity status, the average treatment effect is not nonparametrically identified. To circumvent this, we impose several weak assumptions concerning both the selection and measurement error processes to bound the long-run causal impact of food security. Using data from the ECLS-K and ECLS-B, we assess the identifying power of these assumptions to determine what can be learned about the average treatment effect. While there are a host of interesting findings, two main results arise. First, under the assumption of no measurement error, there exists some evidence that food insecurity has a long-run, negative causal effect on child obesity and overweight status. Second, measurement error is extremely consequential. If only one percent of the households

misreport their food insecurity status, we find only one situation where it is possible to sign the ATE given the selection and measurement error assumptions imposed. 


\section{References}

[1] Alaimo, K., C. Olson, and E. Frongillo (2002), "Family Food Insufficiency, but not Low Family Income, is Positively Associated with Dysthymia and Suicide Symptoms in Adolescents," Journal of Nutrition, $132,719-725$.

[2] Bhargava, A., D. Jolliffe, and L. Howard (2008), "Socioeconomic, Behavioural and Environmental Factors Predicted Body Weights and Household Food Insecurity Scores in the Early Childhood Longitudinal Study-Kindergarten," British Journal of Nutrition, 100, 438-44.

[3] Bhattacharya, J., J. Currie, and S. Haider (2004), "Poverty, Food Insecurity, and Nutritional Outcomes in Children and Adults," Journal of Health Economics, 23, 839-862.

[4] Black, D.A., M.C. Berger, and F.A. Scott (2000), "Bounding Parameter Estimates with Nonclassical Measurement Error," Journal of the American Statistical Association, 95, 739-748

[5] Bound, J., C. Brown, and N. Mathiowetz (2001), "Measurement Error in Survey Data," in E.E. Leamer and J.J. Heckman (eds.) Handbook of Econometrics, Vol. 5, 3705-3843.

[6] Brisbois, T.D., A.P. Farmer, and L.J. McCargar (2012), "Early Markers of Adult Obesity: A Review," Obesity Reviews, 13, 347-367.

[7] Bronte-Tinkew J., M. Zaslow, R. Capps, A. Horowitz, and M. McNamara (2007), "Food Insecurity Works through Depression, Parenting, and Infant Feeding to Influence Overweight and Health in Toddlers," Journal of Nutrition, 137, 2160-2165.

[8] Case, A., D. Lubotsky, and C. Paxson (2002), "Economic Status and Health in Childhood: The Origins of the Gradient," American Economic Review, 92, 1308-1334.

[9] Casey, P., P. Simpson, J. Gossett, M. Bogle, C. Champagne, C. Connell, D. Harsha, B. McCabeSellers, J. Robbins, J. Stuff, and J. Weber (2006), "The Association of Child and Household Food Insecurity with Childhood Overweight Status," Pediatrics, 118, e1406-e1413.

[10] Cawley, J. (2010), "The Economics of Childhood Obesity," Health Affairs, 29, 364-371.

[11] Coleman-Jensen, A., M. Nord, M. Andrews, and S. Carlson (2012), "Household Food Security in the United States in 2011," U.S. Department of Agriculture, Washington, DC. Economic Research Report No. 141. 
[12] Cook, J.T., D.A. Frank, S.M. Levenson, N.B. Neault, T.C. Heeren, M.M. Black, C. Berkowitz, P.H. Casey, A.F. Meyers, D.B. Cutts, and M. Chilton (2006), "Child Food Insecurity Increases Risks Posed by Household Food Insecurity to Young Children's Health," Journal of Nutrition, 136, 1073-1076.

[13] Cook, J.T., D.A. Frank, C. Berkowitz, M.M. Black, P.H. Casey, D.B. Cutts, A.F. Meyers, N. Zaldivar, A. Skalicky, S. Levenson, T. Heeren, and M. Nord (2004), "Food Insecurity is Associated with Adverse Health Outcomes among Human Infants and Toddlers," Journal of Nutrition, 134, 1348-1432.

[14] Dietz, W.H. (1997), "Periods of Risk in Childhood for the Development of Adult Obesity-What Do We Need to Learn?" Journal of Nutrition, 127, 1884S-1886S

[15] Dubois, L., A. Farmer, M. Girard, and M. Porcherie (2006), "Family Food Insufficiency is Related to Overweight Among Preschoolers," Social Science E Medicine, 63, 1503-1516.

[16] Eicher-Miller, H.A., A. Mason, C. Weaver, G. McCabe, C. Boushey (2009), "Food Insecurity is Associated with Iron Deficiency Anemia in U.S. Adolescents," American Journal of Clinical Nutrition, 90, 1358-1371.

[17] Fiese, B.H., C. Gundersen, B. Koester, and L. Washington (2011), "Household Food Insecurity - Serious Concerns for Child Development," Social Policy Report, 25, ISSN 1075-7031 (http://www.srcd.org/spr.html).

[18] Finkelstein, E.A., I.C. Fiebelkorn, and G. Wang (2004), "State-Level Estimates of Annual Medical Expenditures Attributable to Obesity," Obesity Research, 12.

[19] Forshee, R., P. Anderson, P., and M. Storey (2004), "The Role of Beverage Consumption, Physical Activity, Sedentary Behavior, and Demographics on Body Mass Index of Adolescents," International Journal of Food Sciences and Nutrition, 55, 463-478.

[20] Gundersen, C., S. Garasky, and B.J. Lohman (2009), "Food Insecurity is not Associated with Childhood Obesity as Assessed using Multiple Measures of Obesity," Journal of Nutrition, 139, 1173-8.

[21] Gundersen, C., B.J. Lohman, S. Garasky, S.D. Stewart, and J.C. Eisenmann (2008), "Food Security, Maternal Stressors, and Overweight among Low-income US Children: Results from National Health and Nutrition Examination Survey (1999-2002)," Pediatrics, 122, e529-e540.

[22] Gundersen, C. (2013), "Food Insecurity is an Ongoing National Concern," Advances in Nutrition, 4, $36-41$. 
[23] Gundersen, C. and B. Kreider (2008), "Food Stamps and Food Insecurity: What Can Be Learned in the Presence of Nonclassical Measurement Error?" Journal of Human Resources, 43, 352-382.

[24] Gundersen, C. and B. Kreider (2009), "Bounding the Effects of Food Insecurity on Children's Health Outcomes," Journal of Health Economics, 28, 971-983.

[25] Gundersen, C. and D. Ribar (2011), "Food Insecurity and Insufficiency at Low Levels of Food Expenditures," Review of Income and Wealth, 57, 704-726.

[26] Gundersen, C., B. Kreider, J. Pepper (2011), "The Economics of Food Insecurity in the United States," Applied Economic Perspectives and Policy, 33, 281-303.

[27] Hamelin, A., M. Beaudry, and J. Habicht (2002), "Characterization of Household Food Insecurity in Quebec: Food and Feelings," Social Science and Medicine, 54, 119-132.

[28] Howard, L. (2011), "Does Food Insecurity at Home Affect Non-cognitive Performance at School? A Longitudinal Analysis of Elementary Student Classroom Behavior," Economics of Education Review, $30,157-176$.

[29] Huang, J., K. Matta Oshima, and Y. Kim (2010), "Does Food Insecurity Affect Parental Characteristics and Child Behavior? Testing Mediation Effects," Social Service Review, 84, 381-401.

[30] Jimenez-Cruz, A., M. Bacardi-Gascon, and A. Spindler (2003), "Obesity and Hunger among MexicanIndian Migrant Children on the US-Mexico Border," International Journal of Obesity, 27, 740-747.

[31] Johnson, A.D. and C.M. Herbst (2013), "Can We Trust Parental Reports of Child Care Subsidy Receipt?" Children and Youth Services Review, forthcoming

[32] Jyoti, D.F., E.A. Frongillo, and S.J. Jones (2005), “Food Insecurity Affects School Children's Academic Performance, Weight Gain, and Social Skills," Journal of Nutrition, 135, 2831-2839.

[33] Kuku, O., S. Garasky, and C. Gundersen (2012), "The Relationshp between Childhood Obesity and Food Insecurity: A Nonparametric Analysis," Applied Economics, 44, 2667-2677.

[34] Kreider, B. and J. Pepper (2007), "Disability and Employment: Reevaluating the Evidence in Light of Reporting Errors," Journal of the American Statistical Association, 102, 432-441

[35] Kreider, B. and J. Pepper (2008), "Inferring Disability Status from Corrupt Data," Journal of Applied Econometrics, 23, 329-349 
[36] Kreider, B. and S. Hill (2009), "Partially Identifying Treatment Effects with an Application to Covering the Uninsured," Journal of Human Resources, 44, 409-449

[37] Kreider, B., J. Pepper, C. Gundersen, and D. Jolliffe (2012), "Identifying the Effects of SNAP (Food Stamps) on Child Health Outcomes When Participation is Endogenous and Misreported," Journal of the American Statistical Association, 107, 958-975

[38] Liping, P., H.M. Blanck, B. Sherry, K. Dalenius, and L.M. Grummer-Strawn (2012), "Trends in the Prevalence of Extreme Obesity Among US Preschool-Aged Children Living in Low-Income Families, 1998-2010," The Journal of the American Medical Association, 308, 2563-2565.

[39] Manski, C. (1995), "Identification Problems in the Social Sciences," Cambridge, MA: Harvard University Press.

[40] Manski, C. (1997), "Monotone Treatment Response," Econometrica, 65, 1311-1334.

[41] Manski, C. and J. Pepper (2000), "Monotone Instrumental Variables: With an Application to the Returns to Schooling," Econometrica, 68, 997-1010.

[42] Martin, K. and A. Ferris (2007), "Food Insecurity and Gender are Risk Factors for Obesity," Journal of Nutrition Education and Behavior, 39, 31-36.

[43] Martorell, R., A.D. Stein, and D.G. Schroeder (2001), "Early Nutrition and Later Adiposity," Journal of Nutrition, 131, 874S-880S.

[44] Matheson, D., J. Varady, A. Varady, J. Killen (2002), "Household Food Security and Nutritional Status of Hispanic Children in the Fifth Grade," American Journal of Clinical Nutrition, 76, 210-17.

[45] Metallinos-Katsaras, E., A. Must, and K. Gorman (2012), "A Longitudinal Study of Food Insecurity on Obesity in Preschool Children," Journal of the Academy of Nutrition and Dietetics, 112, 1949 1958.

[46] Millimet, D.L., M. Husain, and R. Tchernis (2010), "School Nutrition Programs and the Incidence of Childhood Obesity," Journal of Human Resources, 2010, 45, 640 - 654.

[47] Millimet, D.L., M. Roy, and R. Tchernis (2012), "Federal Nutrition Programs and Childhood Obesity: Inside the Black Box," Review of Economics of the Household, 10, 1-38.

[48] Millimet, D.L. and R. Tchernis (2013), "Estimating Treatment Effects Without an Exclusion Restriction: With an Application to the School Breakfast Program," Journal of Applied Econometrics, forthcoming. 
[49] Muirhead, V., C. Quiñnonez, R. Figueiredo, and D. Locker (2009), "Oral Health Disparities and Food Insecurity in Working Poor Canadians," Community Dentistry and Oral Epidemiology, 37, 294-304.

[50] Nord, M., M. Andrews, and S. Carlson (2009), "Household Food Security in the United States, 2008," U.S. Department of Agriculture, Washington, DC. Economic Research Report No. 83.

[51] Ogden, C. and M. Carroll (2010), "Prevalence of Obesity Among Children and Adolescents: United States, Trends 1963-1965 Through 2007-2008," June, at http://www.cdc.gov/nchs/data/hestat/obesity_child_07_08/obesity_child_07_08.pdf.

[52] Rose, D. and J.N. Bodor (2006), "Household Food Insecurity and Overweight Status in Young School Children: Results from the Early Childhood Longitudinal Study," Pediatrics, 117, 464-473.

[53] Rosin, O. (2008), "The Economic Causes of Obesity: A Survey," Journal of Economic Surveys, 22, $617-647$.

[54] Roy, M. (2012), "Identifying the Effect of WIC on Infant Health When Participation is Endogenous and Misreported," unpublished manuscript, Southern Methodist University.

[55] Schanzenbach, D.W. (2009), "Do School Lunches Contribute to Childhood Obesity?" Journal of Human Resources, 44, 684-709.

[56] Serdula, M.K., D. Ivery, R.J. Coates, D.S. Freedman, D.F. Williamson, and T. Byers (1993), "Do Obese Children Become Obese Adults? A Review of the Literature," Preventative Medicine, 22, 167-177.

[57] Tamer, E. (2010), "Partial Identification in Econometrics," Annual Review of Economics, 2, 167-195.

[58] Van den Berg, G.J., P. Pinger, and J. Schoch (2011), "Instrumental Variable Estimation of the Causal Effect of Hunger Early in Life on Health Later in Life," IZA DP No. 6110.

[59] Walpole, S.C., D. Prieto-Merino, P. Edwards, J. Cleland, G. Stevens, and I. Roberts (2012), "The Weight of Nations: An Estimation of Adult Human Biomass," BMC Public Health, 12, 439.

[60] Whitaker, R.C., S. Phillips, S. Orzol (2006), "Food Insecurity and the Risks of Depression and Anxiety in Mothers and Behavior Problems in their Preschool-aged Children," Pediatrics, 118, 859-868.

[61] World Health Organization (1998), "Obesity: Preventing and Managing the Global Epidemic," WHO Technical Report 894. 


\section{Appendix}

Table A1. Core Food Security Module (CFSM)

1. "We worried whether our food would run out before we got money to buy more". Was that often, sometimes or never true for you in the last 12 months?

2. "The food that we bought just didn’t last and we didn't have money to get more". Was that often, sometimes or never true for you in the last 12 months?

3. "We couldn’t afford to eat balanced meals". Was that often, sometimes or never true for you in the last 12 months?

4. "We relied on only a few kinds of low-cost food to feed our children because we were running out of money to buy food". Was that often, sometimes or never true for you in the last 12 months?

5. In the last 12 months, did you or other adults in the household ever cut the size of your meals or skip meals because there wasn't enough money for food? (Yes/No)

6. "We couldn’t feed our children a balanced meal, because we couldn't afford that". Was that often, sometimes or never true for you in the last 12 months?

7. In the last 12 months, did you ever eat less than you felt you should because there wasn't enough money for food? (Yes/No)

8. (If yes to Question 5) How often did this happen - almost every month, some months but not every month, or in only 1 or 2 months?

9. "The children were not eating enough because we just couldn’t afford enough food". Was that often, sometimes or never true for you in the last 12 months?

10. In the last 12 months, were you ever hungry, but didn't eat, because you couldn't afford enough food? (Yes/No)

11. In the last 12 months, did you lose weight because you didn't have enough money for food? (Yes/No)

12. In the last 12 months, did you ever cut the size of any of the children's meals because there wasn't enough money for food? (Yes/No)

13. In the last 12 months did you or other adults in your household ever not eat for a whole day because there wasn't enough money for food? (Yes/No)

14. In the last 12 months, were the children ever hungry but you just couldn't afford more food? (Yes/No)

15. (If yes to Question 13) How often did this happen - almost every month, some months but not every month, or in only 1 or 2 months?

16. In the last 12 months, did any of the children ever skip a meal because there wasn't enough money for food? (Yes/No)

17. (If yes to Question 16) How often did this happen - almost every month, some months but not every month, or in only 1 or 2 months?

18. In the last 12 months did any of the children ever not eat for a whole day because there wasn't enough money for food? (Yes/No)

Note: Responses in bold are "affirmative". Table taken from Kuku et al. (2012) 


\begin{tabular}{|c|c|c|c|c|c|c|c|c|c|}
\hline \multirow[b]{2}{*}{ Variable } & \multirow[b]{2}{*}{$\mathbf{N}$} & \multirow[b]{2}{*}{ Mean } & \multirow[b]{2}{*}{ SD } & \multicolumn{2}{|c|}{$\begin{array}{c}\text { Marginally Food } \\
\text { Secure } \\
\text { (F1 - FS) }\end{array}$} & \multicolumn{2}{|c|}{ Low Food Security } & \multicolumn{2}{|c|}{$\begin{array}{l}\text { Very Low Food } \\
\text { Security } \\
\text { (F1 - FS) }\end{array}$} \\
\hline & & & & Mean & p-value & Mean & p-value & Mean & p-value \\
\hline Marginally Food Secure (1 = Yes) & 6470 & 0.192 & 0.394 & & & & & & \\
\hline Low Food Security $(1=$ Yes $)$ & 6470 & 0.099 & 0.299 & & & & & & \\
\hline Very Low Food Security (1 = Yes) & 6470 & 0.019 & 0.138 & & & & & & \\
\hline Obese (1 = Yes) & 6470 & 0.242 & 0.428 & 0.043 & 0.006 & 0.021 & 0.301 & 0.049 & 0.277 \\
\hline Overweight ( 1 = Yes) & 6470 & 0.432 & 0.495 & 0.037 & 0.007 & 0.026 & 0.137 & 0.037 & 0.334 \\
\hline SNAP recipient (1 = Yes) & 6470 & 0.153 & 0.360 & 0.242 & 0.000 & 0.281 & 0.000 & 0.313 & 0.000 \\
\hline Household SES Status & 6470 & -0.251 & 0.549 & -0.371 & 0.000 & -0.456 & 0.000 & -0.514 & 0.000 \\
\hline Household Size & 6470 & 4.583 & 1.431 & 0.473 & 0.000 & 0.660 & 0.000 & 0.732 & 0.000 \\
\hline Two Parent Household (1 = Yes) & 6470 & 0.781 & 0.414 & -0.141 & 0.000 & -0.136 & 0.000 & -0.168 & 0.000 \\
\hline One Parent Household (1 = Yes) & 6470 & 0.198 & 0.399 & 0.140 & 0.000 & 0.143 & 0.000 & 0.173 & 0.000 \\
\hline Number of Siblings & 6470 & 1.492 & 1.182 & 0.453 & 0.000 & 0.628 & 0.000 & 0.651 & 0.000 \\
\hline \multicolumn{10}{|l|}{ Mother's Education } \\
\hline Less than High School (1 = Yes) & 6370 & 0.152 & 0.359 & 0.162 & 0.000 & 0.216 & 0.000 & 0.226 & 0.000 \\
\hline High School (1 = Yes) & 6370 & 0.369 & 0.482 & 0.005 & 0.721 & -0.015 & 0.468 & -0.013 & 0.771 \\
\hline Voc. Degree/Some Coll. $(1=$ Yes $)$ & 6370 & 0.373 & 0.484 & -0.086 & 0.000 & -0.103 & 0.000 & -0.124 & 0.005 \\
\hline Bachelor's Degree (1 = Yes) & 6370 & 0.095 & 0.293 & -0.077 & 0.000 & -0.091 & 0.000 & -0.086 & 0.002 \\
\hline Advanced Degree (1 = Yes) & 6370 & 0.011 & 0.104 & -0.005 & 0.174 & -0.007 & 0.107 & -0.004 & 0.699 \\
\hline
\end{tabular}

Notes: Number of observations rounded to the nearest 10 per NCES restricted data requirement. SES = socioeconomic status. Sample excludes households in the highest quintile of SES. Data are from from the kindergarten wave except for obese and overweight which are from the spring fifth grade wave. Omitted category for family structure is 'other or missing', and for mother's education is 'missing'. Columns 5, 7, and 9 report the mean difference between food insecure (F1) households (according to the definition indicated) and food secure (FS) households; p-values obtained using t-test. 


\begin{tabular}{|c|c|c|c|c|c|c|c|c|c|}
\hline \multirow[b]{2}{*}{ Variable } & \multirow[b]{2}{*}{$\mathbf{N}$} & \multirow[b]{2}{*}{ Mean } & \multirow[b]{2}{*}{ SD } & \multicolumn{2}{|c|}{$\begin{array}{c}\text { Marginally Food } \\
\text { Secure } \\
\text { (F1 - FS) }\end{array}$} & \multicolumn{2}{|c|}{ Low Food Security } & \multicolumn{2}{|c|}{$\begin{array}{l}\text { Very Low Food } \\
\text { Security } \\
\text { (F1 - FS) }\end{array}$} \\
\hline & & & & Mean & p-value & Mean & p-value & Mean & p-value \\
\hline Marginally Food Secure (1 = Yes) & 4100 & 0.133 & 0.339 & & & & & & \\
\hline Low Food Security (1 = Yes) & 4100 & 0.128 & 0.334 & & & & & & \\
\hline Very Low Food Security (1 = Yes) & 4100 & 0.034 & 0.181 & & & & & & \\
\hline Obese (1 = Yes) & 4100 & 0.171 & 0.377 & 0.000 & 0.992 & -0.028 & 0.221 & 0.067 & 0.105 \\
\hline Overweight ( 1 = Yes) & 4100 & 0.346 & 0.476 & 0.000 & 0.995 & -0.011 & 0.556 & 0.061 & 0.059 \\
\hline SNAP recipient (1 = Yes) & 4100 & 0.268 & 0.443 & 0.113 & 0.000 & 0.276 & 0.000 & 0.343 & 0.000 \\
\hline Household SES Status & 4100 & -0.376 & 0.584 & -0.247 & 0.000 & -0.446 & 0.000 & -0.461 & 0.000 \\
\hline Household Size & 4100 & 4.561 & 1.561 & 0.158 & 0.028 & 0.184 & 0.011 & 0.179 & 0.176 \\
\hline Two Parent Household (1 = Yes) & 4100 & 0.748 & 0.434 & -0.048 & 0.017 & -0.115 & 0.000 & -0.224 & 0.000 \\
\hline One Parent Household (1 = Yes) & 4100 & 0.244 & 0.430 & 0.050 & 0.012 & 0.118 & 0.000 & 0.232 & 0.000 \\
\hline Number of Siblings & 4100 & 1.140 & 1.165 & 0.106 & 0.048 & 0.208 & 0.000 & 0.385 & 0.000 \\
\hline \multicolumn{10}{|l|}{ Mother's Education } \\
\hline Less than High School (1 = Yes) & 4100 & 0.230 & 0.421 & 0.103 & 0.000 & 0.187 & 0.000 & 0.104 & 0.003 \\
\hline High School (1 = Yes) & 4100 & 0.335 & 0.472 & 0.005 & 0.824 & 0.040 & 0.077 & 0.071 & 0.082 \\
\hline Voc. Degree/Some Coll. $(1=$ Yes $)$ & 4100 & 0.321 & 0.467 & -0.017 & 0.420 & -0.116 & 0.000 & -0.067 & 0.097 \\
\hline Bachelor's Degree (1 = Yes) & 4100 & 0.098 & 0.298 & -0.081 & 0.000 & -0.108 & 0.000 & -0.099 & 0.000 \\
\hline Advanced Degree (1 = Yes) & 4100 & 0.015 & 0.121 & -0.009 & 0.120 & -0.003 & 0.593 & -0.009 & 0.396 \\
\hline
\end{tabular}

Notes: Number of observations rounded to the nearest 50 per NCES restricted data requirement. SES = socioeconomic status.

Sample excludes households in the highest quintile of SES. Data are from from the 9-month wave except for obese and overweight which are from wave 4 (approximately five years old). Omitted category for family structure is 'other or missing', and for mother's education is 'missing'. Columns 5, 7, and 9 report the mean difference between food insecure (F1) households (according to the definition indicated) and food secure (FS) households; p-values obtained using t-test. 
Table A4. Sharp Bounds on the ATE of Low and Marginal Food Security on Child Obesity Status: ECLS-K

\begin{tabular}{|c|c|c|c|c|c|c|c|c|c|c|}
\hline \multirow{3}{*}{$Q$} & \multicolumn{2}{|c|}{ Exogenous Selection } & \multicolumn{2}{|c|}{ No Assumption on Selection } & \multicolumn{2}{|c|}{ MTS } & \multicolumn{2}{|c|}{ MTS \& MIV } & \multicolumn{2}{|c|}{ MTS \& MTR } \\
\hline & (1) & (2) & (3) & (4) & (5) & (6) & (7) & (8) & (9) & (10) \\
\hline & $\mathbf{A E}$ & No $\mathbf{F P}$ & AE & No FP & $\mathbf{A E}$ & No FP & $\mathbf{A E}$ & No FP & $\mathbf{A E}$ & No FP \\
\hline
\end{tabular}

\section{Low Food Security}

0.00 [ $-0.069,0.097$ p.e. $[-0.005,0.098]$ p.e. $\quad[-0.299,0.721]$ p.e. $[-0.299,0.721]$ p.e. $\quad[-0.299,0.097]$ p.e. $\quad[-0.299,0.098]$ p.e. $\quad[-0.308,0.063]$ p.e. [ -0.299 , 0.063] p.e. [ 0.000, 0.097] p.e. [ 0.000, 0.098] p.e.

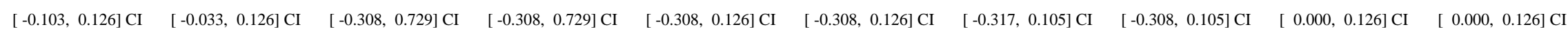

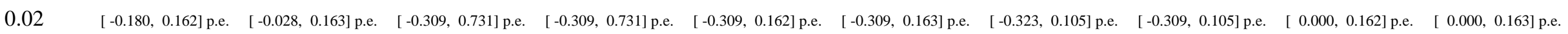

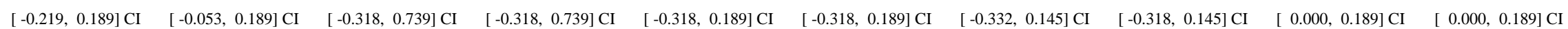

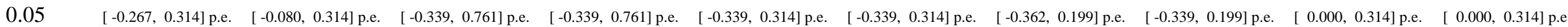
$[-0.276,0.360] \mathrm{CI} \quad[-0.101,0.337] \mathrm{CI} \quad[-0.348,0.769] \mathrm{CI} \quad\left[\begin{array}{l}-0.348,0.769] \mathrm{CI} \\ {[-0.348,0.360] \mathrm{CI}}\end{array} \quad[-0.348,0.337] \mathrm{CI} \quad[-0.372,0.237] \mathrm{CI} \quad[-0.348,0.237] \mathrm{CI} \quad[0.000,0.360] \mathrm{CI} \quad[\quad 0.000,0.337] \mathrm{CI}\right.$

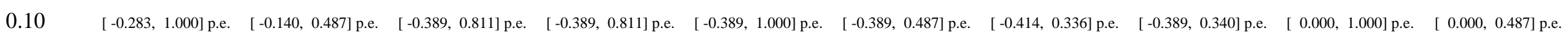
$[-0.293,1.000] \mathrm{CI} \quad[-0.157,0.507] \mathrm{CI} \quad[-0.398,0.819] \mathrm{CI} \quad[-0.398,0.819] \mathrm{CI} \quad[-0.398,1.000] \mathrm{CI} \quad[-0.398,0.507] \mathrm{CI} \quad[-0.424,0.385] \mathrm{CI} \quad[-0.398,0.374] \mathrm{CI} \quad[0.000,1.000] \mathrm{CI} \quad\left[\begin{array}{l}0.000,0.507] \mathrm{CI} \\ \hline\end{array}\right.$

\section{Marginal Food Security}

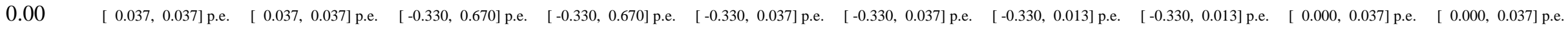

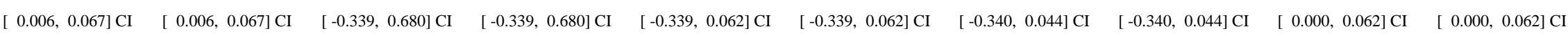

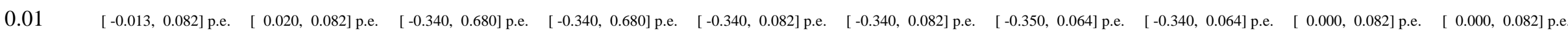

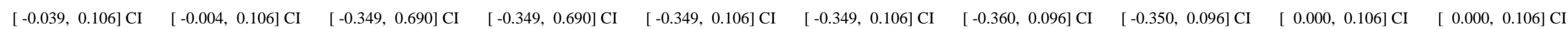

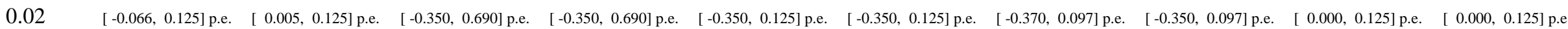
$[-0.095,0.148] \mathrm{CI} \quad[-0.019,0.148] \mathrm{CI} \quad[-0.359,0.700] \mathrm{CI} \quad[-0.359,0.700] \mathrm{CI} \quad[-0.359,0.148] \mathrm{CI} \quad[-0.359,0.148] \mathrm{CI} \quad[-0.379,0.129] \mathrm{CI} \quad[-0.360,0.129] \mathrm{CI} \quad[0.000,0.148] \mathrm{CI} \quad\left[\begin{array}{l}0.000,0.148] \mathrm{CI} \\ \hline\end{array}\right.$

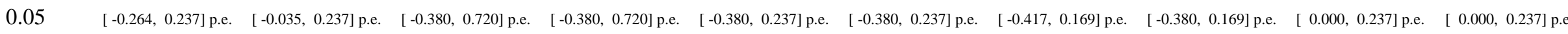

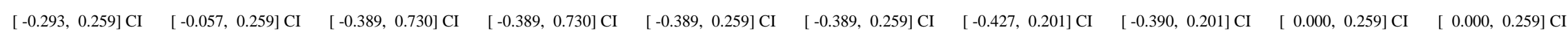

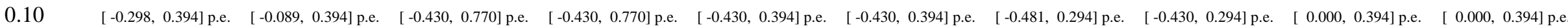

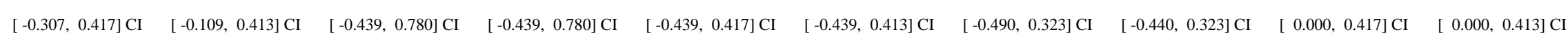

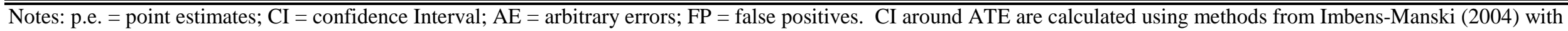
250 pseudosamples. Number of observations $=6470$ (rounded to nearest 10 per NCES restricted data regulations). 
Table A5. Sharp Bounds on the ATE of Low and Marginal Food Security on Child Overweight Status: ECLS-K

\begin{tabular}{|c|c|c|c|c|c|c|c|c|c|c|}
\hline \multirow{3}{*}{$Q$} & \multicolumn{2}{|c|}{ Exogenous Selection } & \multicolumn{2}{|c|}{ "No Assumption on Selection } & \multicolumn{2}{|c|}{ MTS } & \multicolumn{2}{|c|}{ MTS \& MIV } & \multicolumn{2}{|c|}{ MTS \& MTR } \\
\hline & (1) & (2) & (3) & (4) & (5) & (6) & (7) & (8) & (9) & (10) \\
\hline & $\mathbf{A E}$ & No FP & $\mathbf{A E}$ & No FP & AE & No FP & AE & No FP & AE & No FP \\
\hline
\end{tabular}

\title{
I. Low Food Security
}

0.00

$[-0.029,0.058] \mathrm{CI} \quad[-0.029,0.058] \mathrm{CI} \quad[-0.452,0.567] \mathrm{CI} \quad[-0.452,0.567] \mathrm{C}$

0.01

[ $-0.054,0.072]$ p.e. $\quad[-0.031,0.072]$ p.e. $\quad[-0.453,0.567]$ p.e. $\quad[-0.453,0.567]$

$[-0.094,0.107] \mathrm{CI} \quad[-0.064,0.106] \mathrm{CI} \quad[-0.462,0.577] \mathrm{CI} \quad[-0.462,0.577]$

0.02

[ $-0.137,0.136]$ p.e. $\quad[-0.070,0.121]$ p.e. $\quad[-0.463,0.577]$

$[-0.182,0.183] \mathrm{CI} \quad[-0.101,0.152] \mathrm{CI} \quad[-0.472,0.587]$

0.05

0.10

CI $\quad[-0.186,0.261] \mathrm{CI} \quad[-0.502,0.617] \mathrm{C}$

$[-0.494,1.000]$ CI

$-0.262,0.364]$ p.e. $\quad[-0.5$

.284, 0.387] CI

[-0.552, 0.667] C

$[-0.452,0.051] \mathrm{CI} \quad[-0.452,0.051] \mathrm{CI}$

$[-0.444,0.007]$ p.e. $0.000,0.015]$ p.e. $\quad[0.000,0.015]$ p.e.

I. Marginal Food Security

[ 0.043, 0.043] p.

p.e. $\quad[0.043,0.043]$ p.e.

0.01

$[-0.445,0.555]$

$[-0.453,0.072]$ p.e. $\quad[-0.453,0.072]$ p.e. $\quad[-0.464,0.069]$ p.e.

$[-0.454,0.070]$ p.e. $\quad[0.000,0.072]$ p.e. $\quad[0.000,0.072]$ p.e.

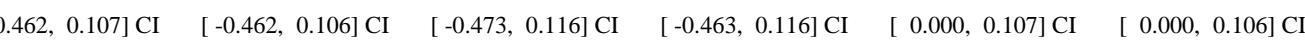

(1)

$[-0.024,0.106]$ CI $\quad[-0.014,0.106]$ CI $\quad[-0.467,0.578]$

0.02

[ $-0.032,0.108]$ p.e. $\quad[-0.011,0.108]$ p.e. $\quad[-0.465,0.575]$

$[-0.065,0.139] \mathrm{CI} \quad[-0.040,0.137] \mathrm{CI} \quad[-0.477,0.588]$

0.05

$[-0.178,0.232]$ p.e. $\quad[-0.081,0.191]$ p.e. $\quad[-0.495,0.605]$

$[-0.216,0.272] \mathrm{CI} \quad[-0.107,0.218] \mathrm{CI} \quad[-0.507,0.618] \mathrm{CI}$

0.10

$[-0.487,0.595]$ p.e. $\quad[-0.176,0.307]$ p.e. $\quad[-0.545,0.655]$ p.e. $\quad[-0.5$

\begin{abstract}
$[-0.445,0.043]$ p.e. $\quad[-0.445,0.043]$ p.e. $\quad[-0.444,0.035]$ p.e. $\quad[-0.444,0.035]$ p.e. $\quad[0.000,0.043]$ p.e. $\quad[0.000,0.043]$ p.e
$[-0.457,0.074] \mathrm{CI} \quad[-0.457,0.074] \mathrm{CI} \quad[-0.457,0.072] \mathrm{CI} \quad[-0.457,0.072] \mathrm{CI} \quad[0.000,0.074] \mathrm{CI} \quad[0.000,0.074] \mathrm{CI}$

$[-0.455,0.565]$ p.e. $\quad[-0.455,0.077]$ p.e. $\quad[-0.455,0.077]$ p.e. $\quad[-0.464,0.072]$ p.e. $\quad[-0.454,0.072]$ p.e. $\quad[0.000,0.077]$ p.e. $\quad[0.000$, 0.077$]$ p.e.

$[-0.467,0.578] \mathrm{CI} \quad[-0.467,0.106] \mathrm{CI} \quad[-0.467,0.106] \mathrm{CI} \quad[-0.477,0.109] \mathrm{CI} \quad[-0.467,0.109] \mathrm{CI} \quad[0.000,0.106] \mathrm{CI} \quad[0.000,0.106] \mathrm{CI}$

$[-0.465,0.575]$ p.e. $\quad[-0.465,0.108]$ p.e. $\quad[-0.465,0.108]$ p.e. $\quad[-0.484,0.104]$ p.e. $\quad[-0.464,0.103]$ p.e. $\quad[0.000,0.108]$ p.e. $\quad[0.000,0.108]$ p.e.

$[-0.495,0.605]$ p.e. $\quad[-0.495,0.232]$ p.e. $\quad[-0.495,0.191]$ p.e. $\quad[-0.541,0.165]$ p.e. $\quad[-0.494,0.154]$ p.e. $\quad[0.000,0.232]$ p.e. $\quad[0.000$, 0.191$]$ p.e.

$[-0.507,0.618] \mathrm{CI} \quad[-0.507,0.272] \mathrm{CI} \quad[-0.507,0.218] \mathrm{CI} \quad[-0.554,0.218] \mathrm{CI} \quad[-0.507,0.194] \mathrm{CI} \quad[0.000,0.272] \mathrm{CI} \quad[0.000,0.218] \mathrm{CI}$
\end{abstract}

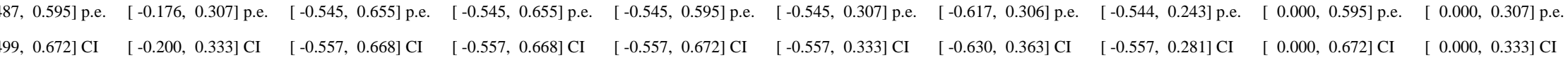

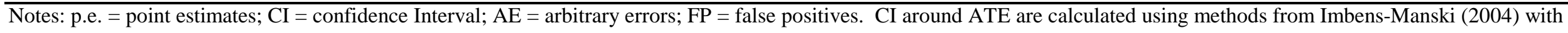
250 pseudosamples. Number of observations $=6470$ (rounded to nearest 10 per NCES restricted data regulations). 
Table A6. Sharp Bounds on the ATE of Low and Marginal Food Security on Child Obesity Status: ECLS-B

\begin{tabular}{|c|c|c|c|c|c|c|c|c|c|c|}
\hline \multirow{3}{*}{$Q$} & \multicolumn{2}{|c|}{ Exogenous Selection } & \multicolumn{2}{|c|}{ No Assumption on Selection } & \multicolumn{2}{|c|}{ MTS } & \multicolumn{2}{|c|}{ MTS \& MIV } & \multicolumn{2}{|c|}{ MTS \& MTR } \\
\hline & (1) & (2) & (3) & (4) & (5) & (6) & (7) & (8) & (9) & (10) \\
\hline & $\mathbf{A E}$ & No FP & $\mathbf{A E}$ & No $\mathbf{F P}$ & $\mathbf{A E}$ & No $\mathbf{F P}$ & $\mathbf{A E}$ & No FP & $\mathbf{A E}$ & No FP \\
\hline
\end{tabular}

\section{Low Food Security}

0.00

$[-0.024,0.036]$ CI $[0.006,0.006]$ p.e. $\quad[-0.276,0.724]$ p.e. $[-0.276,0.724]$

0.01

$[-0.057,0.064]$ p.e. $\quad[-0.006,0.064]$ p.e. $\quad[-0.286,0.734]$

$[-0.084,0.088]$ CI $\quad[-0.030,0.089] \mathrm{CI} \quad[-0.297,0.745] \mathrm{CI} \quad[-0.297,0.745]$

0.02

$[-0.129,0.117]$ p.e. $\quad[-0.017,0.117]$ p.e. $\quad[-0.296,0.744]$

$[-0.157,0.141] \mathrm{CI} \quad[-0.040,0.141] \mathrm{CI} \quad[-0.307,0.755]$

0.05

$[-0.203,0.253]$ p.e. $\quad[-0.046,0.253]$ p.e. $\quad[-0.326,0.774]$ p.e. $\quad[-0.326,0.774]$ p.e

0.10

$0.276]$ CI $\quad[-0.337,0.785]$

$[-0.226,0.454] \mathrm{CI} \quad[-0$.

\section{Marginal Food Security}

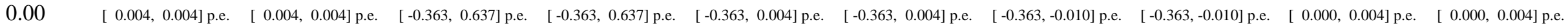
$[-0.020,0.029] \mathrm{CI} \quad[-0.020,0.029] \mathrm{CI} \quad[-0.375,0.649] \mathrm{CI} \quad[-0.375,0.649] \mathrm{CI} \quad[-0.375,0.025] \mathrm{CI} \quad[-0.375,0.025] \mathrm{CI} \quad[-0.376,0.016] \mathrm{CI} \quad[-0.376,0.016] \mathrm{CI} \quad[0.000,0.027] \mathrm{CI} \quad[\quad 0.000,0.027] \mathrm{CI}$

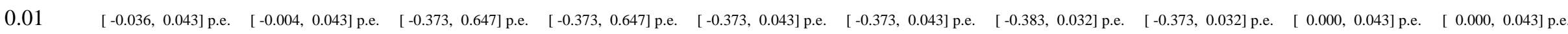

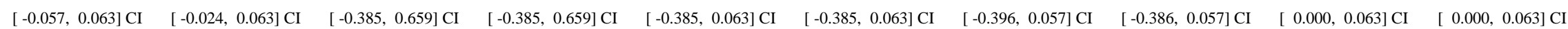

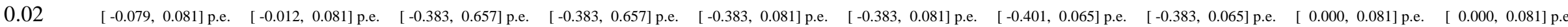
$[-0.100,0.101] \mathrm{CI} \quad[-0.032,0.101] \mathrm{CI} \quad[-0.395,0.669] \mathrm{CI} \quad[-0.395,0.669] \mathrm{CI} \quad[-0.395,0.101] \mathrm{CI} \quad[-0.395,0.101] \mathrm{CI} \quad[-0.414,0.089] \mathrm{CI} \quad[-0.396,0.089] \mathrm{CI} \quad[0.000,0.101] \mathrm{CI} \quad[\quad 0.000,0.101] \mathrm{CI}$

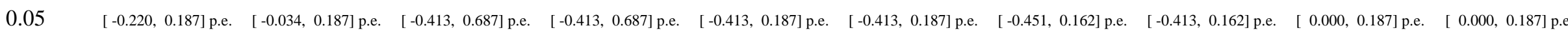
$[-0.237,0.207] \mathrm{CI} \quad[-0.053,0.207] \mathrm{CI} \quad[-0.425,0.699] \mathrm{CI} \quad[-0.425,0.699] \mathrm{CI} \quad[-0.425,0.207] \mathrm{CI} \quad[-0.425,0.207] \mathrm{CI} \quad[-0.463,0.192] \mathrm{CI} \quad[-0.426,0.192] \mathrm{CI} \quad[0.000,0.207] \mathrm{CI} \quad[\quad 0.000,0.207] \mathrm{CI}$

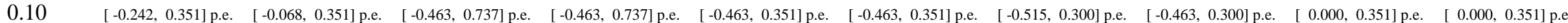
$[-0.255,0.370] \mathrm{CI} \quad[-0.086,0.370] \mathrm{CI} \quad[-0.475,0.749] \mathrm{CI} \quad[-0.475,0.749] \mathrm{CI} \quad[-0.475,0.370] \mathrm{CI} \quad[-0.475,0.370] \mathrm{CI} \quad[-0.527,0.332] \mathrm{CI} \quad[-0.476,0.333] \mathrm{CI} \quad[0.000,0.370] \mathrm{CI} \quad\left[\begin{array}{l}0.000,0.370] \mathrm{CI} \\ \hline\end{array}\right.$

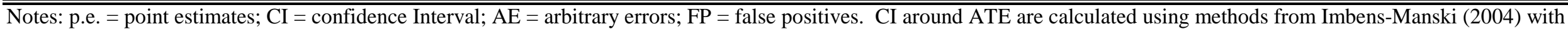
250 pseudosamples. Number of observations $=4100$ (rounded to nearest 50 per NCES restricted data regulations). 
Table A7. Sharp Bounds on the ATE of Low and Marginal Food Security on Child Overweight Status: ECLS-B

\begin{tabular}{|c|c|c|c|c|c|c|c|c|c|c|}
\hline \multirow{3}{*}{$Q$} & \multicolumn{2}{|c|}{ Exogenous Selection } & \multicolumn{2}{|c|}{$\begin{array}{l}\text { No Assumption on Selection } \\
\end{array}$} & \multicolumn{2}{|c|}{ MTS } & \multicolumn{2}{|c|}{ MTS \& MIV } & \multicolumn{2}{|c|}{ MTS \& MTR } \\
\hline & (1) & (2) & (3) & (4) & (5) & (6) & (7) & (8) & (9) & (10) \\
\hline & $\mathbf{A E}$ & No FP & $\mathbf{A E}$ & No FP & $\mathbf{A E}$ & No FP & AE & No FP & AE & No FI \\
\hline
\end{tabular}

\section{Low Food Security}

[ $-0.006,-0.006]$ p.e. $\quad[-0.006,-0.006]$ p.e. $\quad[-0.398,0.602]$ p.e. $\quad[-0.398,0.602]$ p.e. $\quad[-0.398,-0.006]$ p.e. $\quad[-0.398,-0.006]$ p.e. $\quad[-0.397,-0.016]$ p.e. $\quad[-0.397,-0.016]$ p.e. $[-0.044,0.032] \mathrm{CI} \quad[-0.044,0.032] \mathrm{CI} \quad[-0.409,0.614] \mathrm{CI} \quad[-0.409,0.614] \mathrm{CI} \quad[-0.409,0.026] \mathrm{CI} \quad[-0.409,0.026] \mathrm{CI} \quad[-0.408,0.028] \mathrm{CI} \quad[-0.408,0.028] \mathrm{CI}$

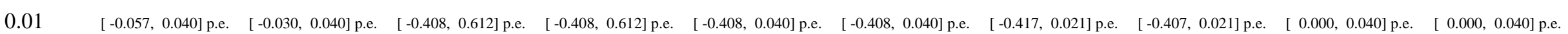

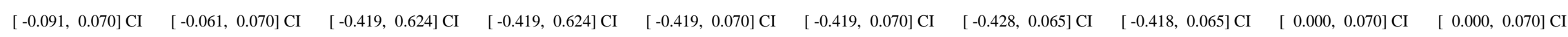

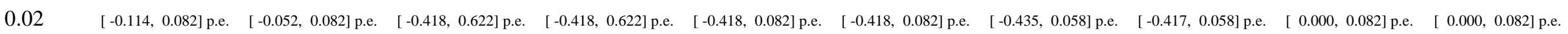
$[-0.150,0.111] \mathrm{CI} \quad[-0.081,0.111] \mathrm{CI} \quad[-0.429,0.634] \mathrm{CI} \quad[-0.429,0.634] \mathrm{CI} \quad[-0.429,0.111] \mathrm{CI} \quad[-0.429,0.111] \mathrm{CI} \quad[-0.447,0.101] \mathrm{CI} \quad[-0.428,0.101] \mathrm{CI} \quad[0.000,0.111] \mathrm{CI} \quad\left[\begin{array}{l}0.000,0.111] \mathrm{CI} \\ \hline\end{array}\right.$

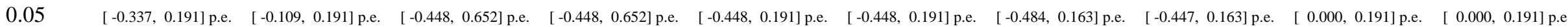

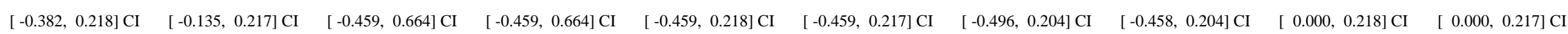

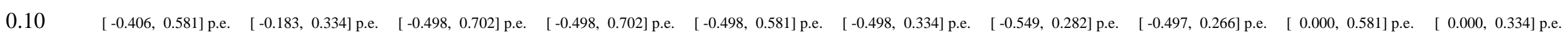

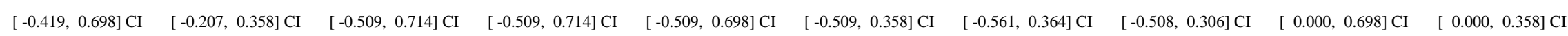

\section{Marginal Food Security}

$0.00 \quad[-0.004,-0.004]$ p.e. $\quad[-0.004,-0.004]$ p.e. $\quad[-0.438,0.562]$ p.e. $\quad[-0.438,0.562]$ p.e. $\quad[-0.438,-0.004]$ p.e. $\quad[-0.438,-0.004]$ p.e. $\quad[-0.439,-0.023]$ p.e. $\quad[-0.439,-0.023]$ p.e. $[-0.037,0.029] \mathrm{CI} \quad[-0.037,0.029] \mathrm{CI} \quad[-0.451,0.575] \mathrm{CI} \quad[-0.451,0.575] \mathrm{CI} \quad[-0.451,0.024] \mathrm{CI} \quad[-0.451,0.024] \mathrm{CI} \quad[-0.451,0.012] \mathrm{CI} \quad[-0.451,0.012] \mathrm{CI}$

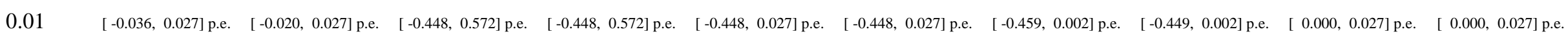
$[-0.064,0.054] \mathrm{CI} \quad[-0.047,0.054] \mathrm{CI} \quad[-0.461,0.585] \mathrm{CI} \quad[-0.461,0.585] \mathrm{CI} \quad[-0.461,0.054] \mathrm{CI} \quad[-0.461,0.054] \mathrm{CI} \quad[-0.471,0.038] \mathrm{CI} \quad[-0.461,0.038] \mathrm{CI} \quad[0.000,0.054] \mathrm{CI} \quad\left[\begin{array}{l}0.000,0.054] \mathrm{CI} \\ \hline\end{array}\right.$

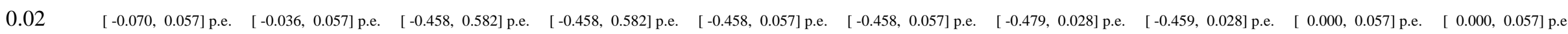
$[-0.098,0.083] \mathrm{CI} \quad[-0.062,0.084] \mathrm{CI} \quad[-0.471,0.595] \mathrm{CI} \quad[-0.471,0.595] \mathrm{CI} \quad[-0.471,0.083] \mathrm{CI} \quad[-0.471,0.084] \mathrm{CI} \quad[-0.491,0.066] \mathrm{CI} \quad[-0.471,0.066] \mathrm{CI} \quad[0.000,0.083] \mathrm{CI} \quad\left[\begin{array}{l}0.000,0.084] \mathrm{CI} \\ \hline\end{array}\right.$

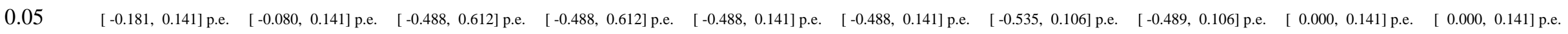
$[-0.213,0.167] \mathrm{CI} \quad[-0.106,0.167] \mathrm{CI} \quad[-0.501,0.625] \mathrm{CI} \quad[-0.501,0.625] \mathrm{CI} \quad[-0.501,0.167] \mathrm{CI} \quad[-0.501,0.167] \mathrm{CI} \quad[-0.548,0.142] \mathrm{CI} \quad[-0.501,0.142] \mathrm{CI} \quad[0.000,0.167] \mathrm{CI} \quad[0.000,0.167] \mathrm{CI}$

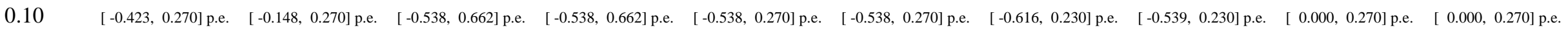

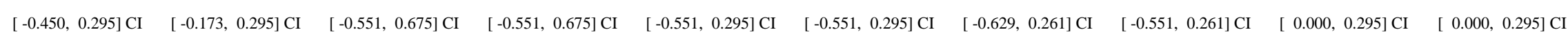

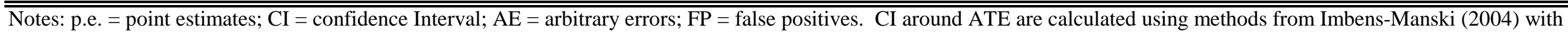

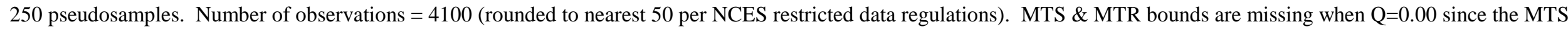
bounds are strictly negative, whereas MTR precludes negative values. 
Table A8. Sharp Bounds on the ATE of Low Food Security with Alternative Control Group: ECLS-K

\begin{tabular}{|c|c|c|c|c|c|c|c|c|c|c|}
\hline \multirow{3}{*}{$Q$} & \multicolumn{2}{|c|}{ Exogenous Selection } & \multicolumn{2}{|c|}{ No Assumption on Selection } & \multicolumn{2}{|c|}{ MTS } & \multicolumn{2}{|c|}{ MTS \& MIV } & \multicolumn{2}{|c|}{ MTS \& MTR } \\
\hline & (1) & (2) & (3) & (4) & (5) & (6) & (7) & (8) & (9) & (10) \\
\hline & $\mathbf{A E}$ & No FP & $\mathbf{A E}$ & No $\mathbf{F P}$ & $\mathbf{A E}$ & No $\mathbf{F P}$ & $\mathbf{A E}$ & No FP & $\mathbf{A E}$ & No FP \\
\hline
\end{tabular}

\section{Obesity}

0.00

[ $0.026,0.026]$ p.e. $\quad[0.026,0.026]$ p.e. $\quad[-0.290,0.710]$ p.e. $\quad[-0.290,0.710]$ p. $[-0.011,0.064] \mathrm{CI} \quad[-0.011,0.064] \mathrm{CI} \quad[-0.299,0.719] \mathrm{CI} \quad[-0.299,0.719] \mathrm{CI}-[-0.299,0.058] \mathrm{CI} \quad[-0.299,0.058] \mathrm{CI} \quad[-0.300,0.041] \mathrm{CI} \quad[-0.300,0.041] \mathrm{CI} \quad[0.000,0.058] \mathrm{CI}-[0.000,0.058] \mathrm{Cl}$

0.01 [ -0.056, 0.097] p.e. $\quad[0.002,0.097]$ p.e. $\quad[-0.300,0.720]$ p.e. $\quad[-0.300,0.720]$ p.e $[-0.091,0.125] \mathrm{CI} \quad[-0.027,0.126] \mathrm{CI} \quad[-0.309,0.729] \mathrm{CI} \quad[-0.309,0.729]$

0.02 [ $-0.155,0.158]$ p.e. $\quad[-0.019,0.158]$ p.e. $\quad[-0.310,0.730]$ $[-0.195,0.185]$ CI $\quad[-0.047,0.185]$ CI $\quad[-0.319,0.739]$ p.e. $\quad[-0.310,0.730]$ $-0.300,0.097]$ p.e. $\quad[-0.300$ [ $0.300,0.097]$ p.e. $\quad[-0.31$ [ $-0.195,0.185] \mathrm{CI} \quad[-0.0$

0.05 [ $-0.265,0.304]$ p.e. $\quad[-0.069,0.304]$ p.e. $\quad[-0.340,0.760]$ p.e. $\quad[-0.340,0.760]$ p.e. $\quad[-0.34$ $[-0.275,0.327] \mathrm{CI} \quad[-0.093,0.327] \mathrm{CI} \quad[-0.349,0.769] \mathrm{CI} \quad[-0.349,0.769] \mathrm{CI} \quad[-0.349,0.327] \mathrm{CI} \quad[-0.34$

0.10 $[-0.280,1.000]$ p.e. $\quad[-0.128,0.476]$ p.e. $\quad[-0.390,0.810]$ p.e. $\quad[-0.390,0.810]$ p.e. $\quad[-0.39$ $[-0.291,1.000] \mathrm{CI} \quad[-0.148,0.496] \mathrm{CI} \quad[-0.399,0.819] \mathrm{CI} \quad[-0.399,0.819] \mathrm{CI} \quad[-0.3$ $[-0.390,1.000]$ p.e. $\quad[-0.390,0.476]$ p.e. $\quad[-0.41$ $-0.399,1.000]$ CI $\quad[-0.399,0.496]$ CI $\quad[-0.4$ $0.311,0.064]$ p.e. $\quad[-0.301,0.064]$ p.e. $\quad[0.000,0.097]$ p.e. $\quad[0.000,0.097]$ p.e. $-0.310,0.158]$ p.e. $\quad[-0.310,0.158]$ p.e. $\quad[-0.326,0.089]$ p.e. $\quad[-0.311,0.089]$ p.e. $\quad[0.000,0.158]$ p.e. $\quad[0.000,0.158]$ p.e. $-0.319,0.185] \mathrm{CI} \quad[-0.319,0.185] \mathrm{CI} \quad[-0.335,0.133] \mathrm{CI} \quad[-0.320,0.133] \mathrm{CI} \quad[0.000,0.185] \mathrm{CI} \quad[0.000,0.185] \mathrm{CI}$

\section{Overweight}

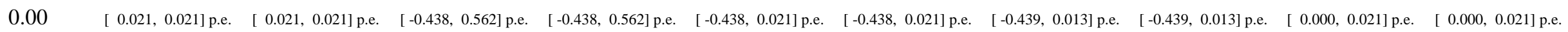
$[-0.019,0.062] \mathrm{CI} \quad[-0.019,0.062] \mathrm{CI} \quad[-0.449,0.574] \mathrm{CI} \quad[-0.449,0.574] \mathrm{CI} \quad[-0.449,0.056] \mathrm{CI} \quad[-0.449,0.056] \mathrm{CI} \quad[-0.450,0.061] \mathrm{CI} \quad[-0.450,0.061] \mathrm{CI} \quad[0.000,0.056] \mathrm{CI} \quad[\quad 0.000,0.056] \mathrm{CI}$

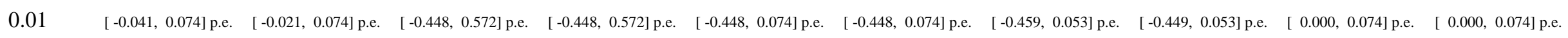
$[-0.077,0.108] \mathrm{CI} \quad[-0.052,0.107] \mathrm{CI} \quad[-0.459,0.584] \mathrm{CI} \quad[-0.459,0.584] \mathrm{CI} \quad[-0.459,0.108] \mathrm{CI} \quad[-0.459,0.107] \mathrm{CI} \quad[-0.470,0.102] \mathrm{CI} \quad[-0.460,0.102] \mathrm{CI} \quad[0.000,0.108] \mathrm{CI} \quad\left[\begin{array}{l}0.000,0.107] \mathrm{CI} \\ \hline\end{array}\right.$

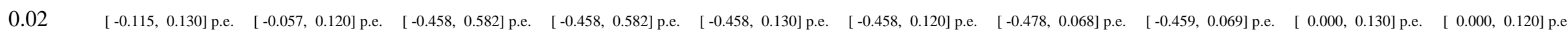
$[-0.155,0.172] \mathrm{CI} \quad[-0.086,0.151] \mathrm{CI} \quad[-0.469,0.594] \mathrm{CI} \quad[-0.469,0.594] \mathrm{CI} \quad[-0.469,0.172] \mathrm{CI} \quad[-0.469,0.151] \mathrm{CI} \quad[-0.490,0.123] \mathrm{CI} \quad[-0.470,0.121] \mathrm{CI} \quad[0.000,0.172] \mathrm{CI} \quad\left[\begin{array}{l}0.000,0.151] \mathrm{CI} \\ \hline\end{array}\right.$

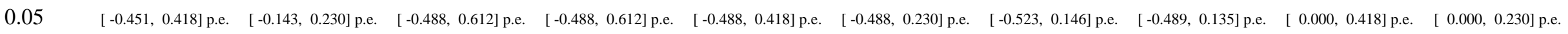
$[-0.483,0.498] \mathrm{CI} \quad[-0.168,0.258] \mathrm{CI} \quad[-0.499,0.624] \mathrm{CI} \quad[-0.499,0.624] \mathrm{CI} \quad[-0.499,0.498] \mathrm{CI} \quad[-0.499,0.258] \mathrm{CI} \quad[-0.535,0.213] \mathrm{CI} \quad[-0.500,0.183] \mathrm{CI} \quad[\quad 0.000,0.498] \mathrm{CI} \quad[\quad 0.000,0.258] \mathrm{CI}$

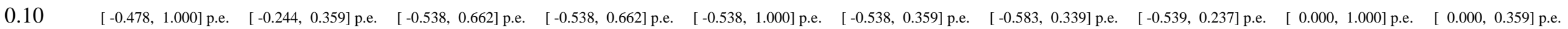
$[-0.489,1.000] \mathrm{CI} \quad[-0.266,0.384] \mathrm{CI} \quad[-0.549,0.674] \mathrm{CI} \quad[-0.549,0.674] \mathrm{CI} \quad[-0.549,1.000] \mathrm{CI} \quad[-0.549,0.384] \mathrm{CI} \quad[-0.595,0.430] \mathrm{CI} \quad[-0.550,0.279] \mathrm{CI} \quad[0.000,1.000] \mathrm{CI} \quad\left[\begin{array}{l}0.000,0.384] \mathrm{CI} \\ \hline\end{array}\right.$

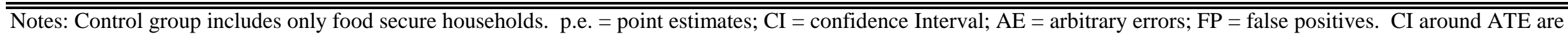

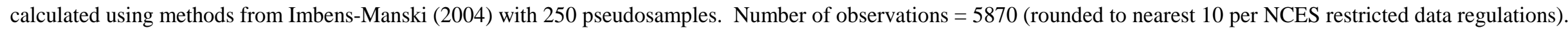


Table A9. Sharp Bounds on the ATE of Low Food Security with Alternative Control Group: ECLS-B

\begin{tabular}{|c|c|c|c|c|c|c|c|c|c|c|}
\hline \multirow{3}{*}{$Q$} & \multicolumn{2}{|c|}{ Exogenous Selection } & \multicolumn{2}{|c|}{ "No Assumption on Selection } & \multicolumn{2}{|c|}{ MTS } & \multicolumn{2}{|c|}{ MTS \& MIV } & \multicolumn{2}{|c|}{ MTS \& MTR } \\
\hline & (1) & (2) & (3) & (4) & (5) & (6) & (7) & (8) & (9) & (10) \\
\hline & $\mathbf{A E}$ & No FP & $\mathbf{A E}$ & No $\mathbf{F P}$ & $\mathbf{A E}$ & No FP & $\mathbf{A E}$ & No $F P$ & $\mathbf{A E}$ & No $F P$ \\
\hline
\end{tabular}

\section{Obesity}

0.00

[ $0.007,0.007]$ p.e. $\quad[0.007,0.007]$ p.e. $\quad[-0.292,0.708]$ p.e. $\quad[-0.292,0.708]$ p. $[-0.025,0.038] \mathrm{CI} \quad[-0.025,0.038] \mathrm{CI} \quad[-0.304,0.720] \mathrm{CI} \quad[-0.304,0.720] \mathrm{CI} \quad[-0.304,0.033] \mathrm{CI} \quad[-0.304,0.033] \mathrm{CI} \quad[-0.304,0.007] \mathrm{CI} \quad[-0.304,0.007] \mathrm{CI} \quad[0.000,0.036] \mathrm{CI} \quad[0.000,0.036] \mathrm{CI}$

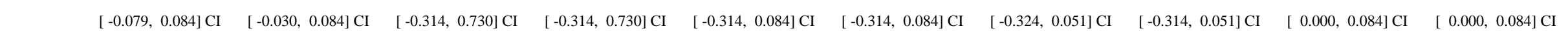

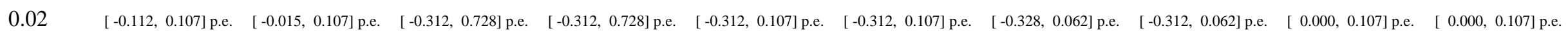
$[-0.143,0.131] \mathrm{CI} \quad[-0.040,0.131] \mathrm{CI} \quad[-0.324,0.740] \mathrm{CI} \quad[-0.324,0.740] \mathrm{CI} \quad[-0.324,0.131] \mathrm{CI} \quad[-0.324,0.131] \mathrm{CI} \quad[-0.340,0.094] \mathrm{CI} \quad[-0.324,0.094] \mathrm{CI} \quad[0.000,0.131] \mathrm{CI} \quad\left[\begin{array}{l}0.000,0.131] \mathrm{CI} \\ \hline\end{array}\right.$

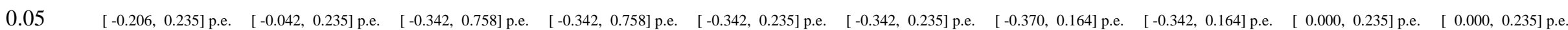

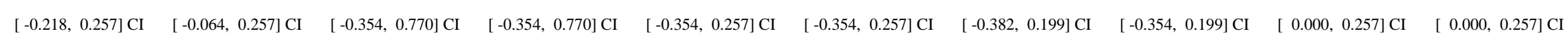

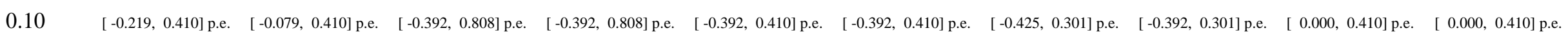
$[-0.233,0.431] \mathrm{CI} \quad[-0.099,0.431] \mathrm{CI} \quad[-0.404,0.820] \mathrm{CI} \quad[-0.404,0.820] \mathrm{CI} \quad[-0.404,0.431] \mathrm{CI} \quad[-0.404,0.431] \mathrm{CI} \quad[-0.438,0.334] \mathrm{CI} \quad[-0.404,0.334] \mathrm{CI} \quad[0.000,0.431] \mathrm{CI} \quad\left[\begin{array}{l}0.000,0.431] \mathrm{CI} \\ \hline\end{array}\right.$

\section{Overweight}

$0.00 \quad[-0.006,-0.006]$ p.e. $[-0.006,-0.006]$ p.e. $[-0.405,0.595]$ p.e. $\quad[-0.405,0.595]$ p.e. $\quad[-0.405,-0.006]$ p.e. $[-0.405,-0.006]$ p.e. $[-0.407,-0.055]$ p.e. $[-0.407,-0.055]$ p.e. $[-0.040,0.028] \mathrm{CI} \quad[-0.040,0.028] \mathrm{CI} \quad[-0.417,0.606] \mathrm{CI} \quad[-0.417,0.606] \mathrm{CI} \quad[-0.417,0.022] \mathrm{CI} \quad[-0.417,0.022] \mathrm{CI} \quad[-0.419,-0.014] \mathrm{CI} \quad[-0.419,-0.014] \mathrm{CI}$

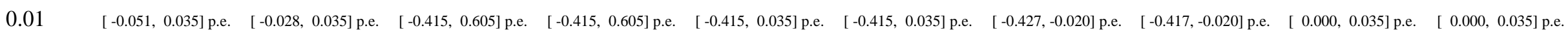
$[-0.081,0.063] \mathrm{CI} \quad[-0.055,0.063] \mathrm{CI} \quad[-0.427,0.616] \mathrm{CI} \quad[-0.427,0.616] \mathrm{CI} \quad[-0.427,0.063] \mathrm{CI} \quad[-0.427,0.063] \mathrm{CI} \quad[-0.439,0.023] \mathrm{CI} \quad[-0.429,0.023] \mathrm{CI} \quad[0.000,0.063] \mathrm{CI} \quad[\quad 0.000,0.063] \mathrm{CI}$

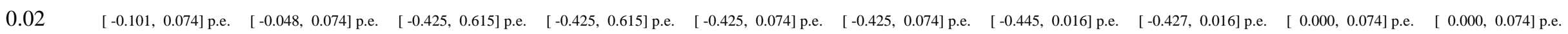
$[-0.132,0.101] \mathrm{CI} \quad[-0.074,0.101] \mathrm{CI} \quad[-0.437,0.626] \mathrm{CI} \quad[-0.437,0.626] \mathrm{CI} \quad[-0.437,0.101] \mathrm{CI} \quad[-0.437,0.101] \mathrm{CI} \quad[-0.457,0.057] \mathrm{CI} \quad[-0.439,0.057] \mathrm{CI} \quad[0.000,0.101] \mathrm{CI} \quad\left[\begin{array}{l}0.000,0.101] \mathrm{CI} \\ \hline\end{array}\right.$

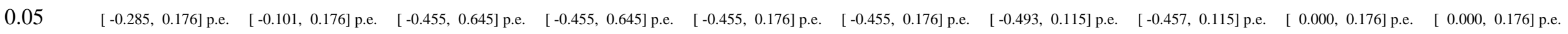
$[-0.325,0.201] \mathrm{CI} \quad[-0.125,0.201] \mathrm{CI} \quad[-0.467,0.656] \mathrm{CI} \quad[-0.467,0.656] \mathrm{CI} \quad[-0.467,0.201] \mathrm{CI} \quad[-0.467,0.201] \mathrm{CI} \quad[-0.505,0.155] \mathrm{CI} \quad[-0.469,0.155] \mathrm{CI} \quad[0.000,0.201] \mathrm{CI} \quad[\quad 0.000,0.201] \mathrm{CI}$

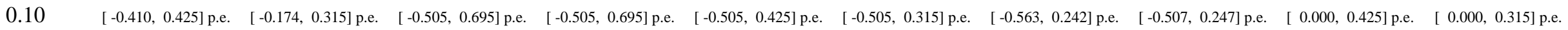

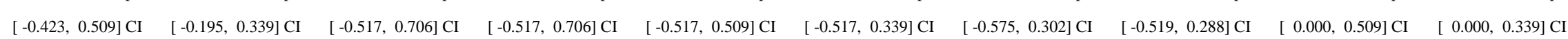

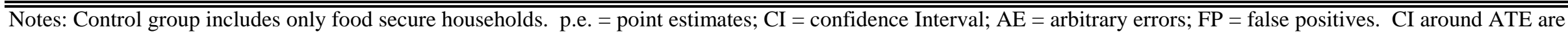

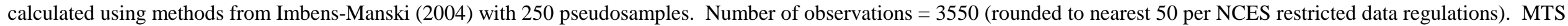
\& MTR bounds are missing when $\mathrm{Q}=0.00$ since the MTS bounds are strictly negative, whereas MTR precludes negative values. 


\section{Figure 1. Sharp Bounds on the ATE of Very Low Food Security on Child Obesity Status: ECLS-K}

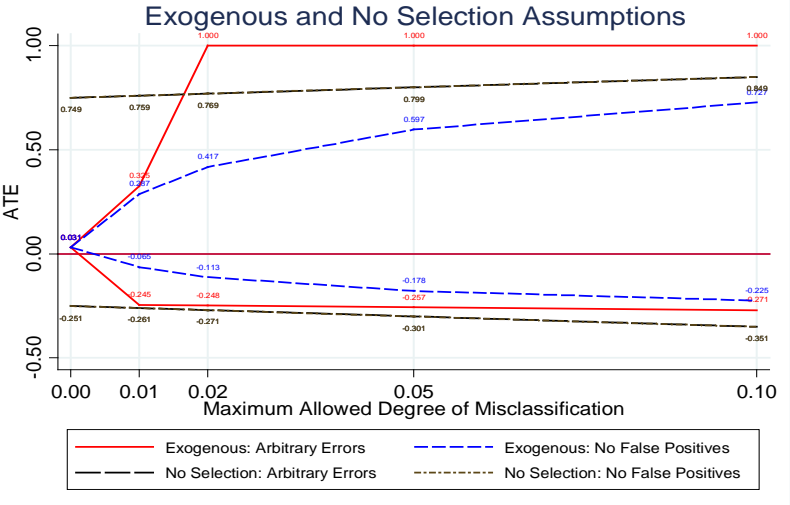

\section{A. Exogenous Selection or No Assumption}

Notes: $\mathrm{AE}=$ arbitrary errors; $\mathrm{FP}=$ false positives.

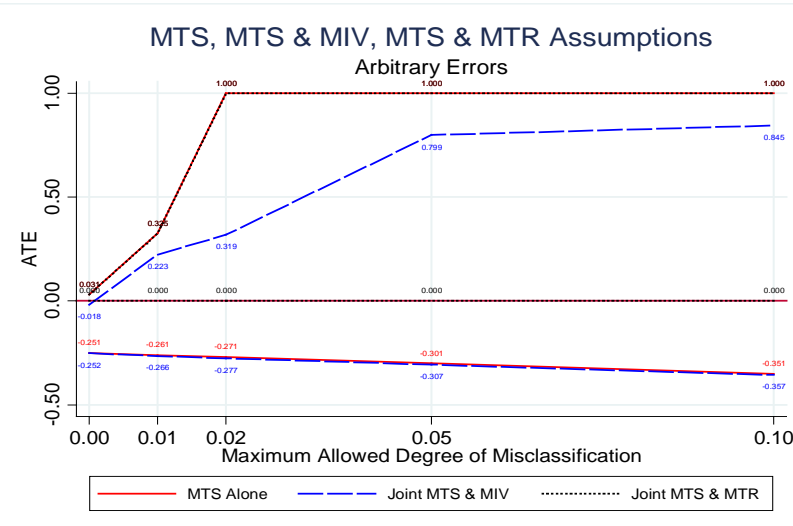

B. Only MTS or MTS-MIV or MTS-MTR: AE

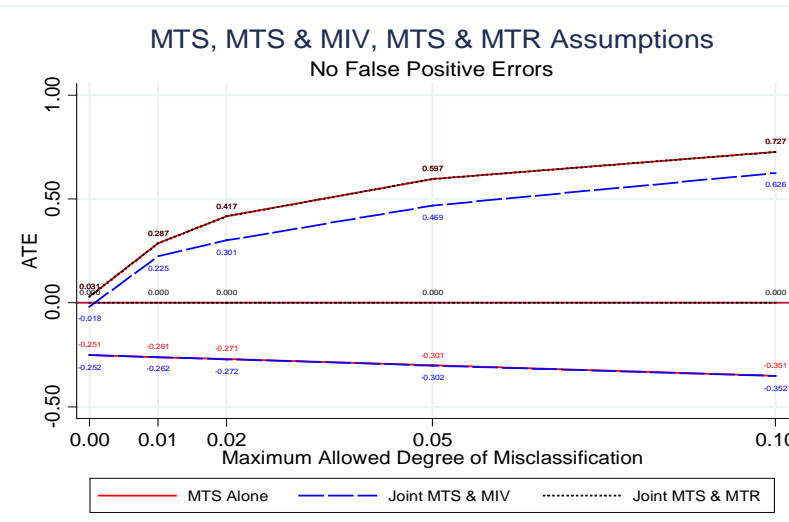

C. Only MTS or MTS-MIV or MTS-MTR: No FP

Table 1. Sharp Bounds on the ATE of Very Low Food Security on Child Obesity Status: ECLS-K

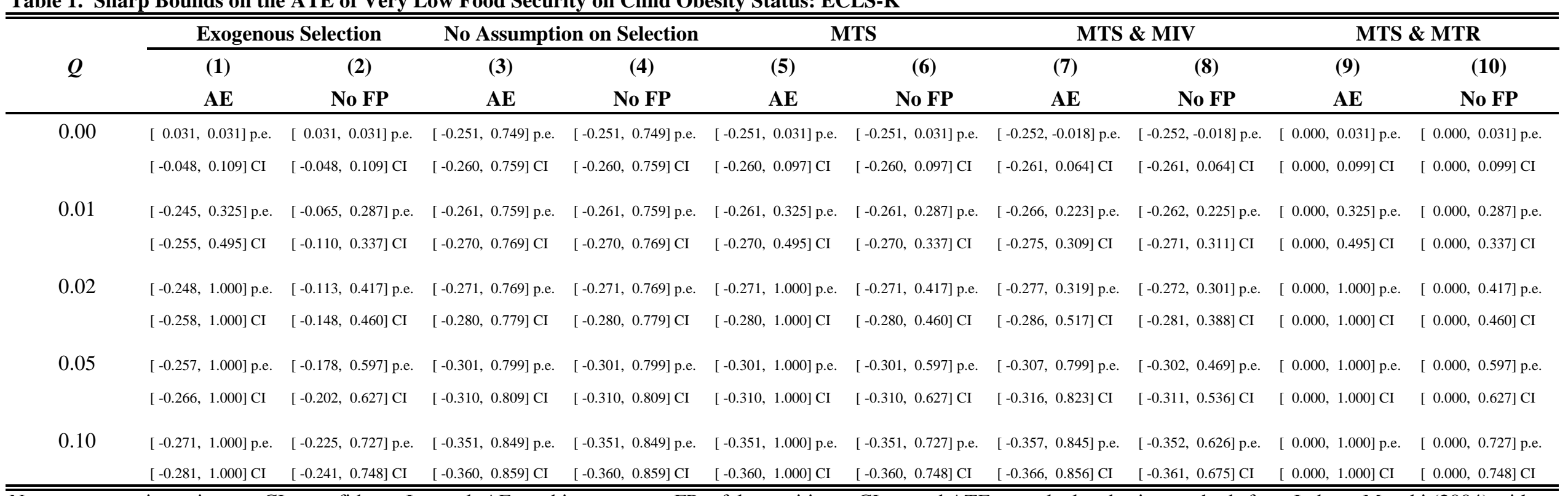

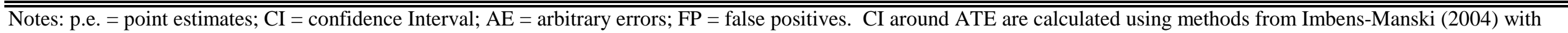
250 pseudosamples. Number of observations $=6470$ (rounded to nearest 10 per NCES restricted data regulations). See Appendix A and text for further details. 
Figure 2. Sharp Bounds on the ATE of Very Low Food Security on Child Overweight Status: ECLS-K

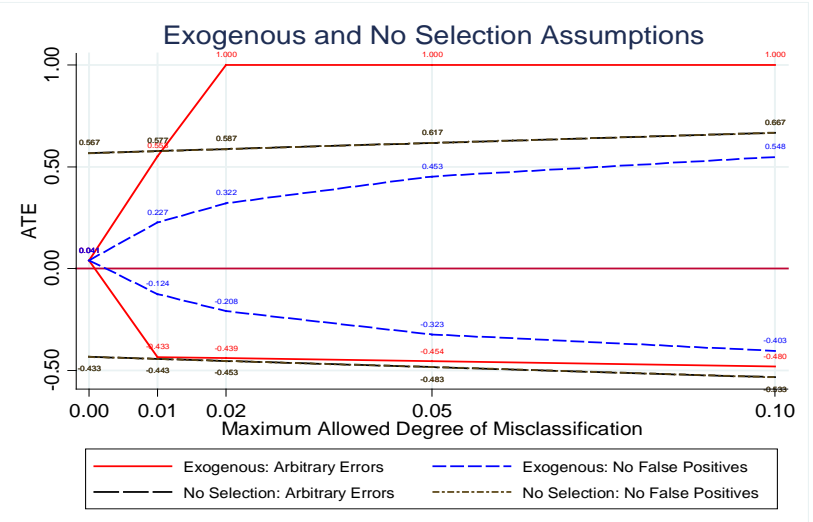

\section{A. Exogenous Selection or No Assumption}

Notes: $\mathrm{AE}=$ arbitrary errors; $\mathrm{FP}=$ false positives.

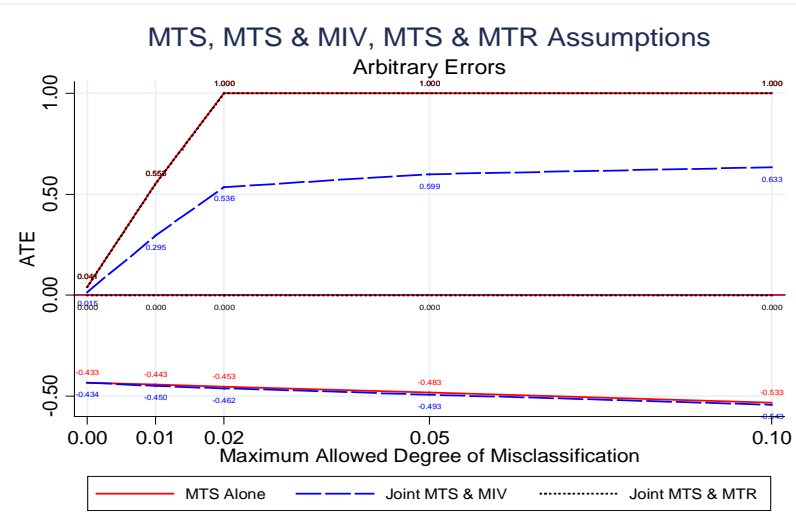

B. Only MTS or MTS-MIV or MTS-MTR: AE

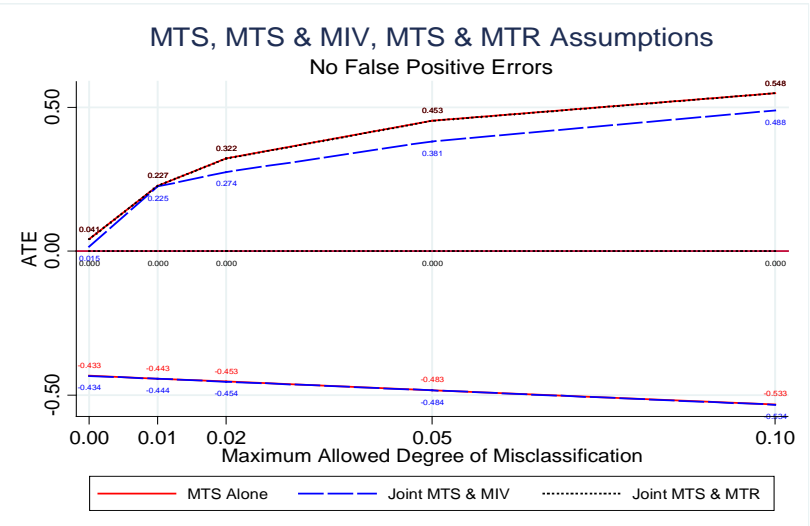

C. Only MTS or MTS-MIV or MTS-MTR: No FP

Table 2. Sharp Bounds on the ATE of Very Low Food Security on Child Overweight Status: ECLS-K

\begin{tabular}{|c|c|c|c|c|c|c|c|c|c|c|}
\hline \multirow{3}{*}{$Q$} & \multicolumn{2}{|c|}{ Exogenous Selection } & \multicolumn{2}{|c|}{ No Assumption on Selection } & \multicolumn{2}{|c|}{ MTS } & \multicolumn{2}{|c|}{ MTS \& MIV } & \multicolumn{2}{|c|}{ MTS \& MTR } \\
\hline & (1) & (2) & (3) & (4) & (5) & (6) & (7) & (8) & (9) & (10) \\
\hline & $\mathbf{A E}$ & No FP & $\mathbf{A E}$ & No FP & AE & No FP & $\mathbf{A E}$ & No FP & $\mathbf{A E}$ & No FP \\
\hline \multirow[t]{2}{*}{0.00} & [ $0.041,0.041]$ p.e. & [ 0.041, 0.041] p.e. & {$[-0.433,0.567]$ p.e. } & {$[-0.433,0.567]$ p.e. } & {$[-0.433,0.041]$ p.e. } & {$[-0.433,0.041]$ p.e. } & {$[-0.434,0.015]$ p.e. } & {$[-0.434,0.015]$ p.e. } & [ $0.000,0.041]$ p.e. & [ $0.000,0.041]$ p.e. \\
\hline & {$[-0.037,0.119] \mathrm{CI}$} & {$[-0.037,0.119] \mathrm{CI}$} & {$[-0.444,0.578] \mathrm{CI}$} & {$[-0.444,0.578] \mathrm{CI}$} & {$[-0.444,0.106]$ CI } & {$[-0.444,0.106] \mathrm{CI}$} & {$[-0.445,0.144] \mathrm{CI}$} & {$[-0.445,0.144] \mathrm{CI}$} & {$[0.000,0.108] \mathrm{CI}$} & {$[0.000,0.108] \mathrm{CI}$} \\
\hline \multirow[t]{2}{*}{0.01} & [ - $0.433,0.553]$ p.e. & {$[-0.124,0.227]$ p.e. } & {$[-0.443,0.577]$ p.e. } & [ -0.443, 0.577] p.e. & {$[-0.443,0.553]$ p.e. } & {$[-0.443,0.227]$ p.e. } & {$[-0.450,0.295]$ p.e. } & {$[-0.444,0.225]$ p.e. } & [ 0.000, 0.553] p.e. & {$[0.000,0.227]$ p.e. } \\
\hline & {$[-0.486,0.781] \mathrm{CI}$} & {$[-0.171,0.275]$ CI } & {$[-0.454,0.588] \mathrm{CI}$} & {$[-0.454,0.588] \mathrm{CI}$} & {$[-0.454,0.781] \mathrm{CI}$} & {$[-0.454,0.275] \mathrm{CI}$} & {$[-0.461,0.455] \mathrm{CI}$} & {$[-0.455,0.305] \mathrm{CI}$} & {$[0.000,0.781] \mathrm{CI}$} & {$[0.000,0.275] \mathrm{CI}$} \\
\hline \multirow[t]{2}{*}{0.02} & [ - $0.439,1.000]$ p.e. & [ - $0.208,0.322]$ p.e. & {$[-0.453,0.587]$ p.e. } & {$[-0.453,0.587]$ p.e. } & {$[-0.453,1.000]$ p.e. } & [ - $0.453,0.322]$ p.e. & [ -0.462, 0.536$]$ p.e. & {$[-0.454,0.274]$ p.e. } & [ $0.000,1.000]$ p.e. & [ $0.000,0.322]$ p.e. \\
\hline & {$[-0.450,1.000] \mathrm{CI}$} & {$[-0.246,0.361] \mathrm{CI}$} & {$[-0.464,0.598] \mathrm{CI}$} & {$[-0.464,0.598] \mathrm{CI}$} & {$[-0.464,1.000] \mathrm{CI}$} & {$[-0.464,0.361] \mathrm{CI}$} & {$[-0.473,0.744] \mathrm{CI}$} & {$[-0.465,0.355] \mathrm{CI}$} & {$[0.000,1.000] \mathrm{CI}$} & {$[0.000,0.361] \mathrm{CI}$} \\
\hline \multirow[t]{2}{*}{0.05} & {$[-0.454,1.000]$ p.e. } & {$[-0.323,0.453]$ p.e. } & {$[-0.483,0.617]$ p.e. } & [ -0.483, 0.617$]$ p.e. & {$[-0.483,1.000]$ p.e. } & {$[-0.483,0.453]$ p.e. } & [ - -0.493, 0.599] p.e. & {$[-0.484,0.381]$ p.e. } & [ $0.000,1.000]$ p.e. & [ $0.000,0.453]$ p.e. \\
\hline & {$[-0.466,1.000] \mathrm{CI}$} & {$[-0.348,0.479] \mathrm{CI}$} & {$[-0.494,0.628]$ CI } & {$[-0.494,0.628] \mathrm{CI}$} & {$[-0.494,1.000]$ CI } & {$[-0.494,0.479] \mathrm{CI}$} & {$[-0.504,0.611]$ CI } & {$[-0.495,0.446] \mathrm{CI}$} & {$[0.000,1.000] \mathrm{CI}$} & {$[0.000,0.479] \mathrm{CI}$} \\
\hline \multirow[t]{2}{*}{0.10} & {$[-0.480,1.000]$ p.e. } & {$[-0.403,0.548]$ p.e. } & {$[-0.533,0.667]$ p.e. } & [ -0.533, 0.667] p.e. & {$[-0.533,1.000]$ p.e. } & [ - $0.533,0.548]$ p.e. & {$[-0.543,0.633]$ p.e. } & {$[-0.534,0.488]$ p.e. } & [ $0.000,1.000]$ p.e. & [ $0.000,0.548]$ p.e. \\
\hline & {$[-0.492,1.000] \mathrm{CI}$} & {$[-0.422,0.568] \mathrm{CI}$} & {$[-0.544,0.678]$ CI } & {$[-0.544,0.678] \mathrm{CI}$} & {$[-0.544,1.000] \mathrm{CI}$} & {$[-0.544,0.568] \mathrm{CI}$} & {$[-0.554,0.645] \mathrm{CI}$} & {$[-0.545,0.534] \mathrm{CI}$} & {$[0.000,1.000] \mathrm{CI}$} & {$[0.000,0.568] \mathrm{CI}$} \\
\hline
\end{tabular}


Figure 3. Sharp Bounds on the ATE of Very Low Food Security on Child Obesity Status: ECLS-B

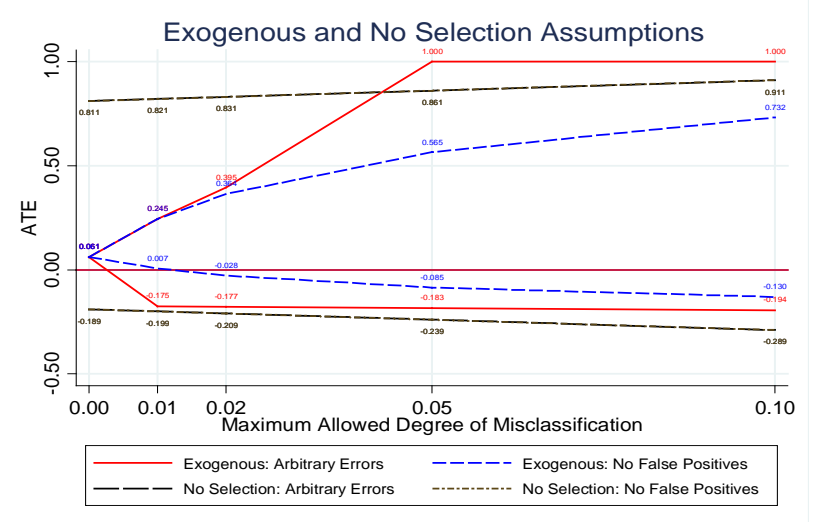

\section{A. Exogenous Selection or No Assumption}

Notes: $\mathrm{AE}=$ arbitrary errors; $\mathrm{FP}=$ false positives.

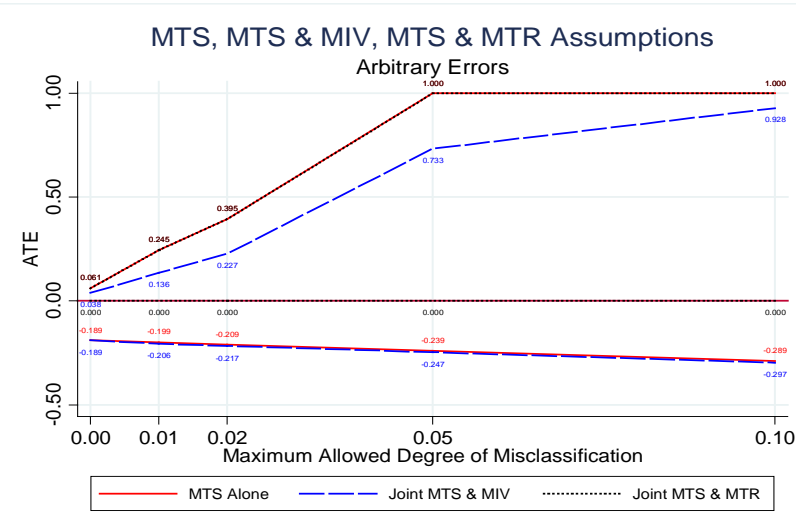

B. Only MTS or MTS-MIV or MTS-MTR: AE

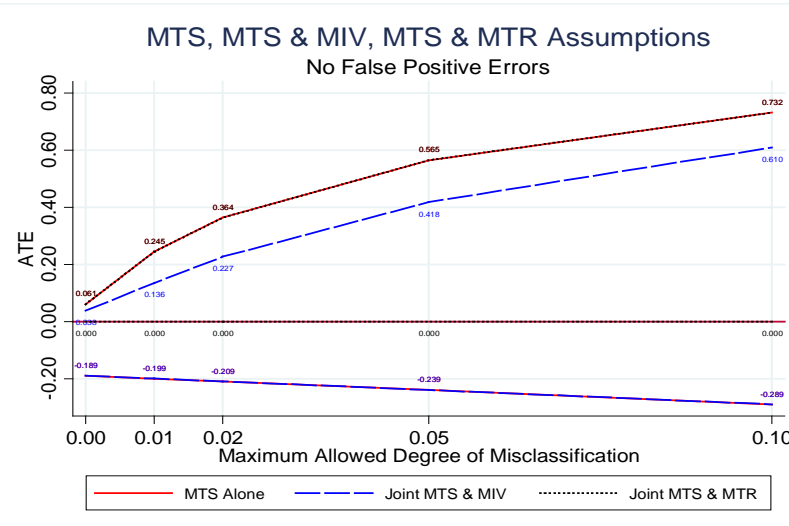

C. Only MTS or MTS-MIV or MTS-MTR: No FP

Table 3. Sharp Bounds on the ATE of Very Low Food Security on Child Obesity Status: ECLS-B

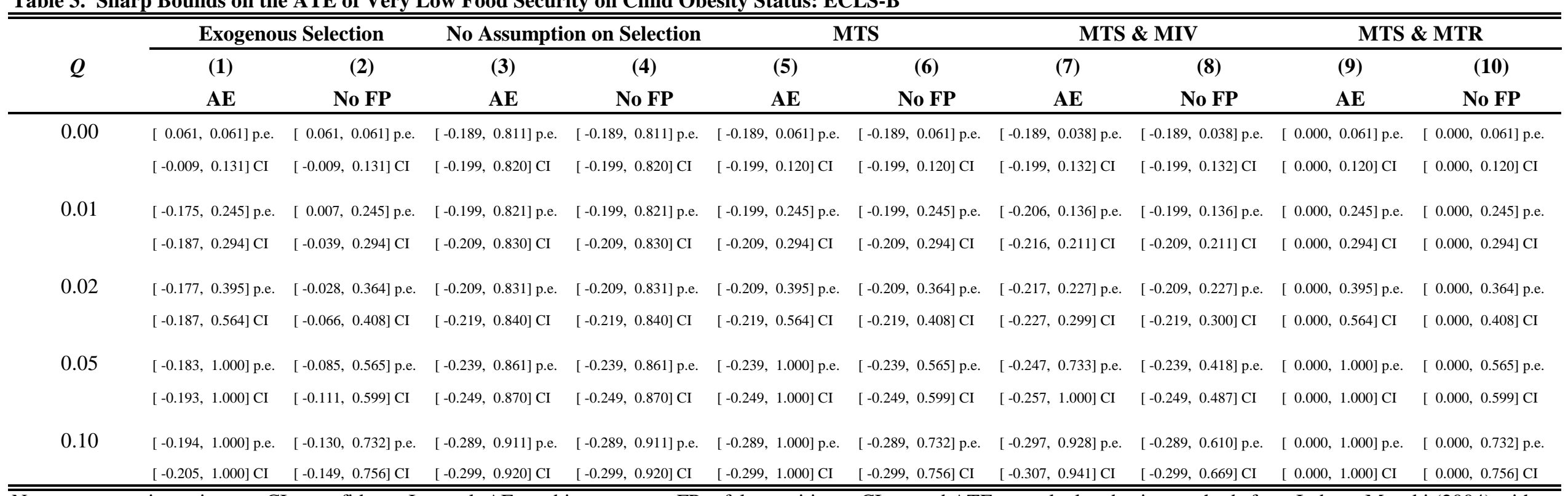

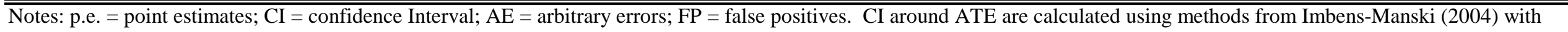
250 pseudosamples. Number of observations $=4100$ (rounded to nearest 50 per NCES restricted data regulations). See Appendix A and text for further details. 


\section{Figure 4. Sharp Bounds on the ATE of Very Low Food Security on Child Overweight Status: ECLS-B}
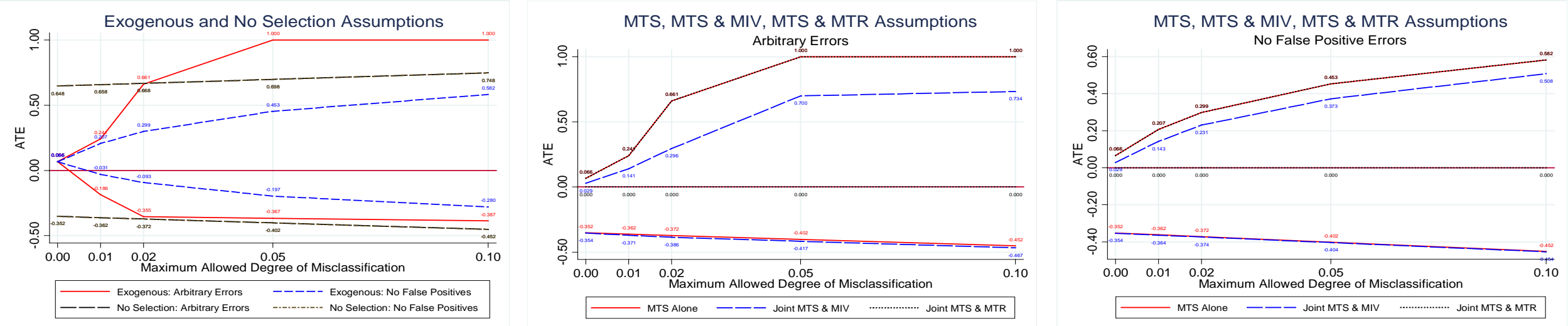

\section{A. Exogenous Selection or No Assumption}

B. Only MTS or MTS-MIV or MTS-MTR: AE

C. Only MTS or MTS-MIV or MTS-MTR: No FP

Notes: $\mathrm{AE}=$ arbitrary errors; $\mathrm{FP}=$ false positives.

Table 4. Sharp Bounds on the ATE of Very Low Food Security on Child Overweight Status: ECLS-B

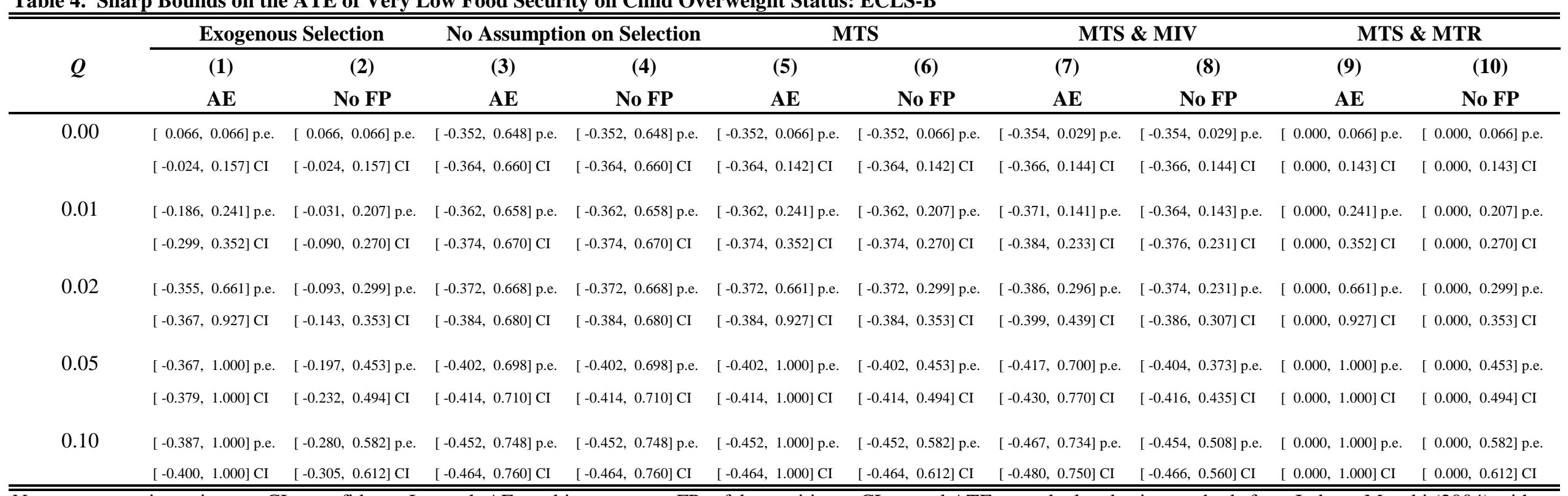

Notes: p.e. = point estimates; $\mathrm{CI}=$ confidence Interval; $\mathrm{AE}=$ arbitrary errors; $\mathrm{FP}=$ false positives. $\mathrm{CI}$ around ATE are calculated using methods from Imbens-Manski (2004) with 250 pseudosamples. Number of observations $=4100$ (rounded to nearest 50 per NCES restricted data regulations). See Appendix A and text for further details. 
Figure 5. Sharp Bounds on the ATE of Very Low Food Security on Child Obesity Status with Alternative Control Group: ECLS-K

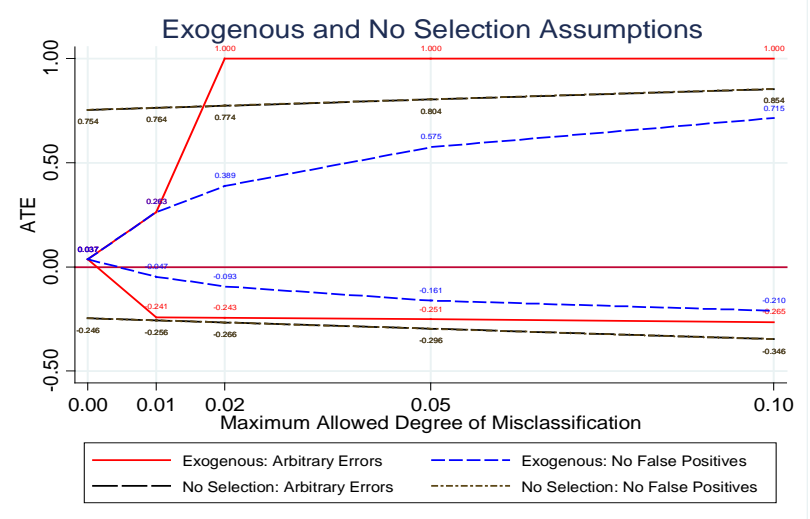

\section{A. Exogenous Selection or No Assumption}

Notes: $\mathrm{AE}=$ arbitrary errors; $\mathrm{FP}=$ false positives.

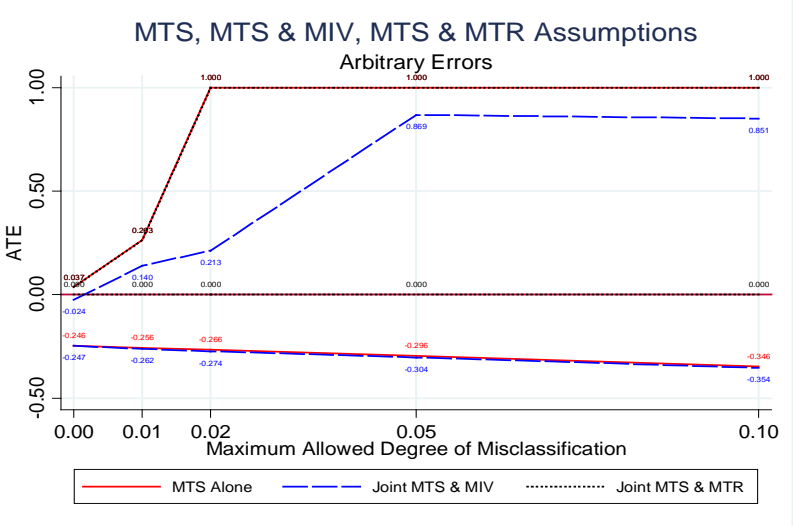

B. Only MTS or MTS-MIV or MTS-MTR: AE

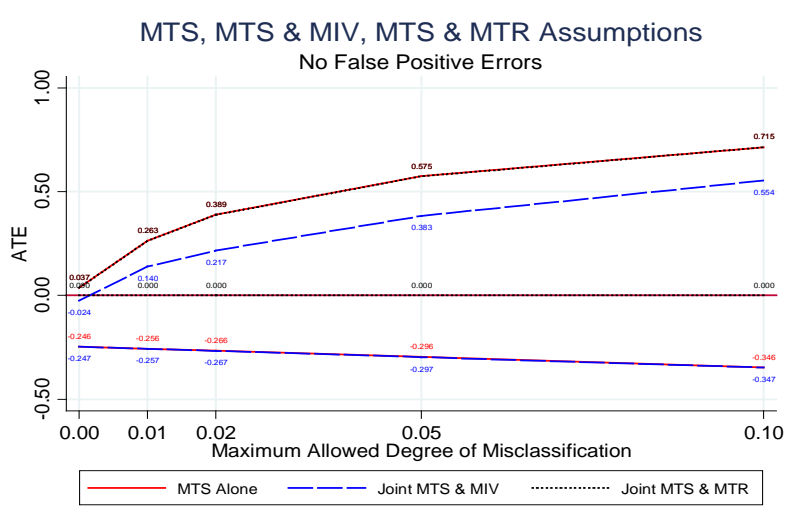

C. Only MTS or MTS-MIV or MTS-MTR: No FP

Table 5. Sharp Bounds on the ATE of Very Low Food Security on Child Obesity Status with Alternative Control Group: ECLS-K

\begin{tabular}{|c|c|c|c|c|c|c|c|c|c|c|c|c|}
\hline \multirow{3}{*}{$Q$} & \multicolumn{2}{|c|}{ Exogenous Selection } & \multicolumn{2}{|c|}{ "No Assumption on Selection } & \multicolumn{3}{|c|}{ MTS } & \multicolumn{2}{|c|}{ MTS \& MIV } & \multicolumn{3}{|c|}{ MTS \& MTR } \\
\hline & (1) & (2) & (3) & (4) & & (5) & (6) & (7) & (8) & & (9) & (10) \\
\hline & $\mathbf{A E}$ & No FP & $\mathbf{A E}$ & No FP & & $\mathbf{A E}$ & No FP & $\mathbf{A E}$ & No FP & & $\mathbf{A E}$ & No FP \\
\hline 0.00 & {$[-0.038,0.112] \mathrm{CI}$} & {$[-0.038,0.112] \mathrm{CI}$} & {$[-0.256,0.763]$ CI } & {$[-0.256,0.763] \mathrm{CI}$} & {$[-0.256$} & $0.100] \mathrm{CI}$ & {$[-0.256,0.100] \mathrm{CI}$} & {$[-0.257,0.053]$ CI } & {$[-0.257,0.053] \mathrm{CI}$} & {$[0.000$} & $0.102] \mathrm{CI}$ & {$[0.000,0.102] \mathrm{CI}$} \\
\hline 0.01 & [ - $0.241,0.263]$ p.e. & {$[-0.047,0.263]$ p.e. } & {$[-0.256,0.764]$ p.e. } & {$[-0.256,0.764]$ p.e. } & {$[-0.256$} & $0.263]$ p.e. & {$[-0.256,0.263]$ p.e. } & {$[-0.262,0.140]$ p.e. } & {$[-0.257,0.140]$ p.e. } & {$[0.000$} & $0.263]$ p.e. & [ $0.000,0.263]$ p.e. \\
\hline \multirow[t]{2}{*}{0.02} & {$[-0.243,1.000]$ p.e. } & {$[-0.093,0.389]$ p.e. } & [ - $0.266,0.774]$ p.e. & {$[-0.266,0.774]$ p.e. } & {$[-0.266$,} & 1.000] p.e. & [ - $0.266,0.389]$ p.e. & {$[-0.274,0.213]$ p.e. } & [ -0.267, 0.217$]$ p.e. & [ 0.000 , & $1.000]$ p.e. & [ $0.000,0.389]$ p.e. \\
\hline & {$[-0.253,1.000] \mathrm{CI}$} & {$[-0.130,0.429] \mathrm{CI}$} & {$[-0.276,0.783] \mathrm{CI}$} & {$[-0.276,0.783] \mathrm{CI}$} & {$[-0.276$} & 1.000] CI & {$[-0.276,0.429] \mathrm{CI}$} & {$[-0.284,0.267]$ CI } & {$[-0.277,0.273] \mathrm{CI}$} & {$[0.000$} & $1.000] \mathrm{CI}$ & {$[0.000,0.429] \mathrm{CI}$} \\
\hline \multirow[t]{2}{*}{0.05} & {$[-0.251,1.000]$ p.e. } & {$[-0.161,0.575]$ p.e. } & {$[-0.296,0.804]$ p.e. } & {$[-0.296,0.804]$ p.e. } & {$[-0.296$,} & 1.000] p.e. & [ -0.296, 0.575$]$ p.e. & {$[-0.304,0.869]$ p.e. } & [ -0.297, 0.383$]$ p.e. & [ 0.000 & 1.000] p.e. & [ $0.000,0.575]$ p.e. \\
\hline & {$[-0.261,1.000] \mathrm{CI}$} & {$[-0.184,0.603] \mathrm{CI}$} & {$[-0.306,0.813] \mathrm{CI}$} & {$[-0.306,0.813] \mathrm{CI}$} & {$[-0.306$,} & 1.000] CI & {$[-0.306,0.603] \mathrm{CI}$} & {$[-0.314,1.000]$ CI } & {$[-0.307,0.428] \mathrm{CI}$} & {$[0.000$} & $1.000] \mathrm{CI}$ & {$[\quad 0.000,0.603] \mathrm{CI}$} \\
\hline 0.10 & {$[-0.265,1.000]$ p.e. } & {$[-0.210,0.715]$ p.e. } & {$[-0.346,0.854]$ p.e. } & {$[-0.346,0.854]$ p.e. } & {$[-0.346$} & $1.000]$ p.e. & {$[-0.346,0.715]$ p.e. } & {$[-0.354,0.851]$ p.e. } & {$[-0.347,0.554]$ p.e. } & {$[0.000$} & 1.000] p.e. & {$[0.000,0.715]$ p.e. } \\
\hline
\end{tabular}

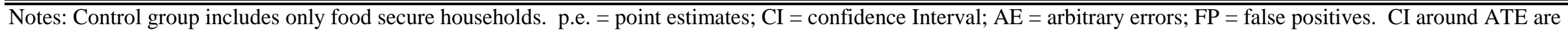

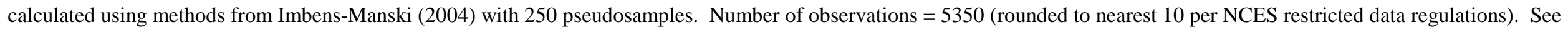
Appendix A and text for further details. 
Figure 6. Sharp Bounds on the ATE of Very Low Food Security on Child Overweight Status with Alternative Control Group: ECLS-K

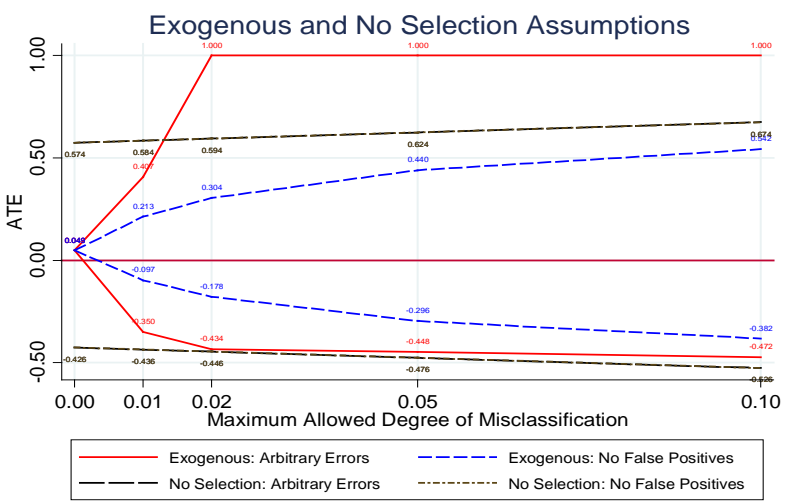

\section{A. Exogenous Selection or No Assumption}

Notes: $\mathrm{AE}=$ arbitrary errors; $\mathrm{FP}=$ false positives.

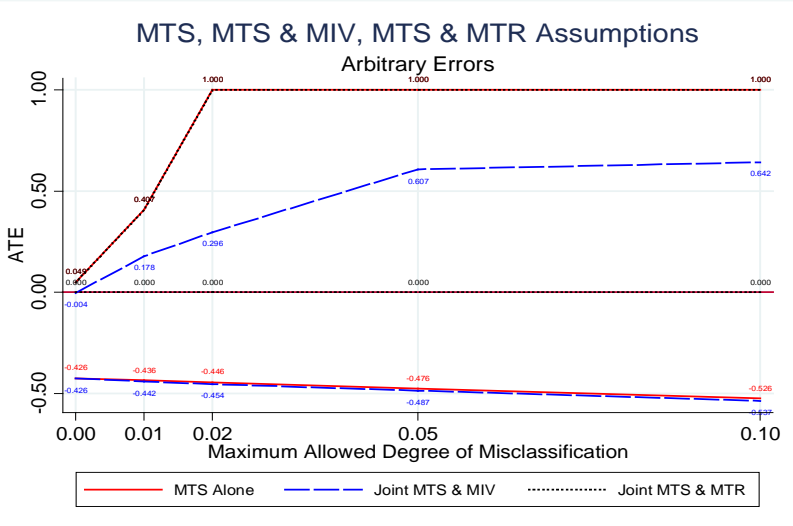

B. Only MTS or MTS-MIV or MTS-MTR: AE

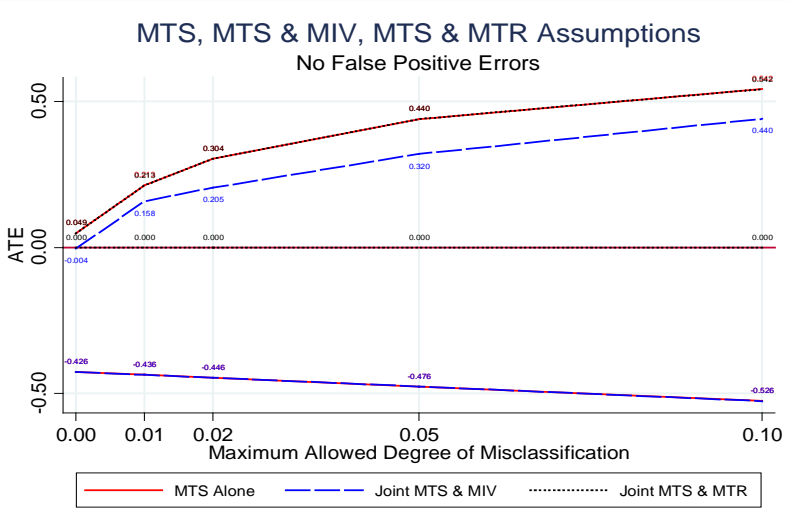

C. Only MTS or MTS-MIV or MTS-MTR: No FP

Table 6. Sharp Bounds on the ATE of Very Low Food Security on Child Overweight Status with Alternative Control Group: ECLS-K

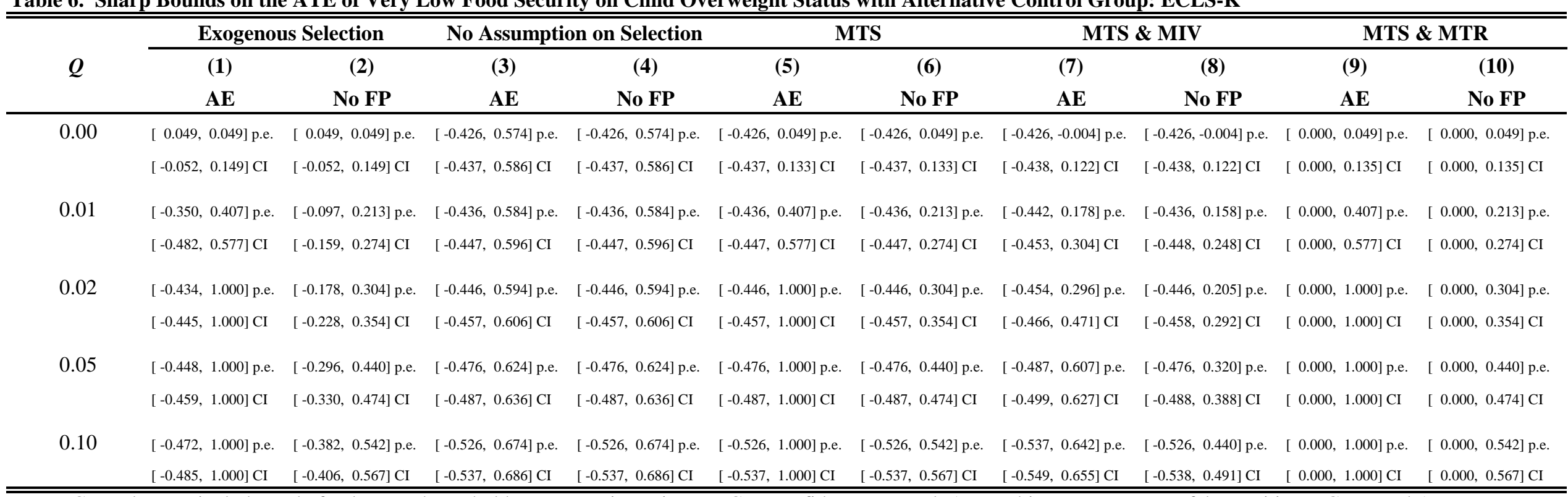

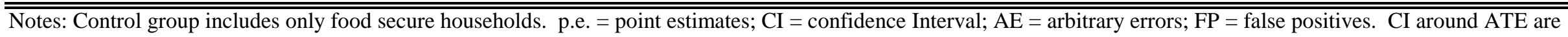

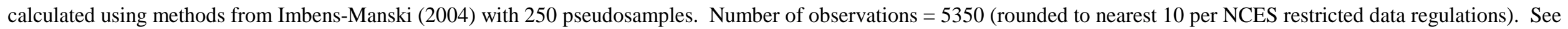
Appendix A and text for further details. 


\section{Figure 7. Sharp Bounds on the ATE of Very Low Food Security on Child Obesity Status with Alternative Control Group: ECLS-B}

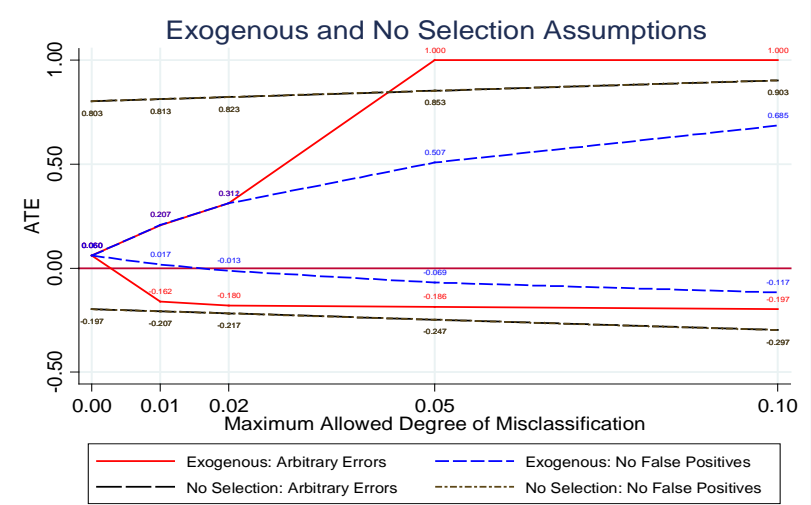

\section{A. Exogenous Selection or No Assumption}

Notes: $\mathrm{AE}=$ arbitrary errors; $\mathrm{FP}=$ false positives.

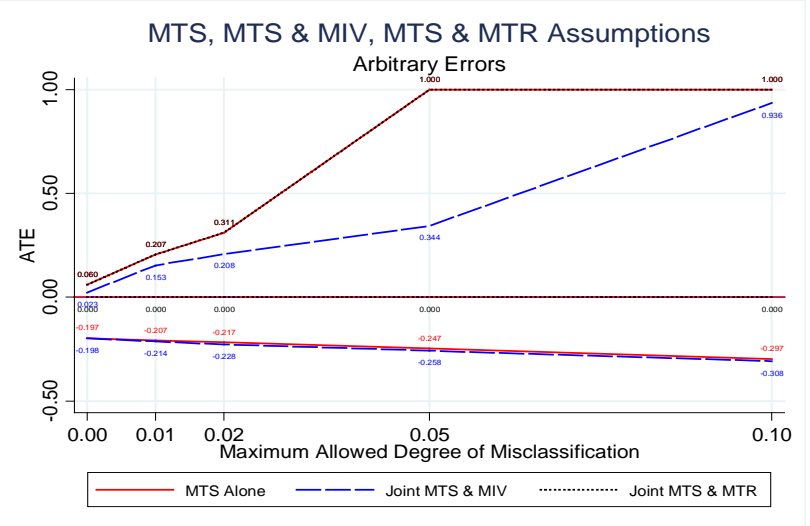

B. Only MTS or MTS-MIV or MTS-MTR: AE

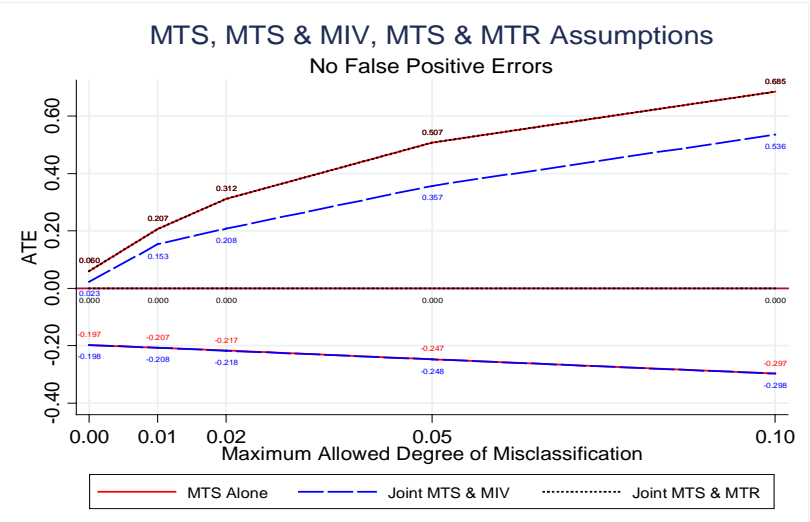

C. Only MTS or MTS-MIV or MTS-MTR: No FP

Table 7. Sharp Bounds on the ATE of Very Low Food Security on Child Obesity Status with Alternative Control Group: ECLS-B

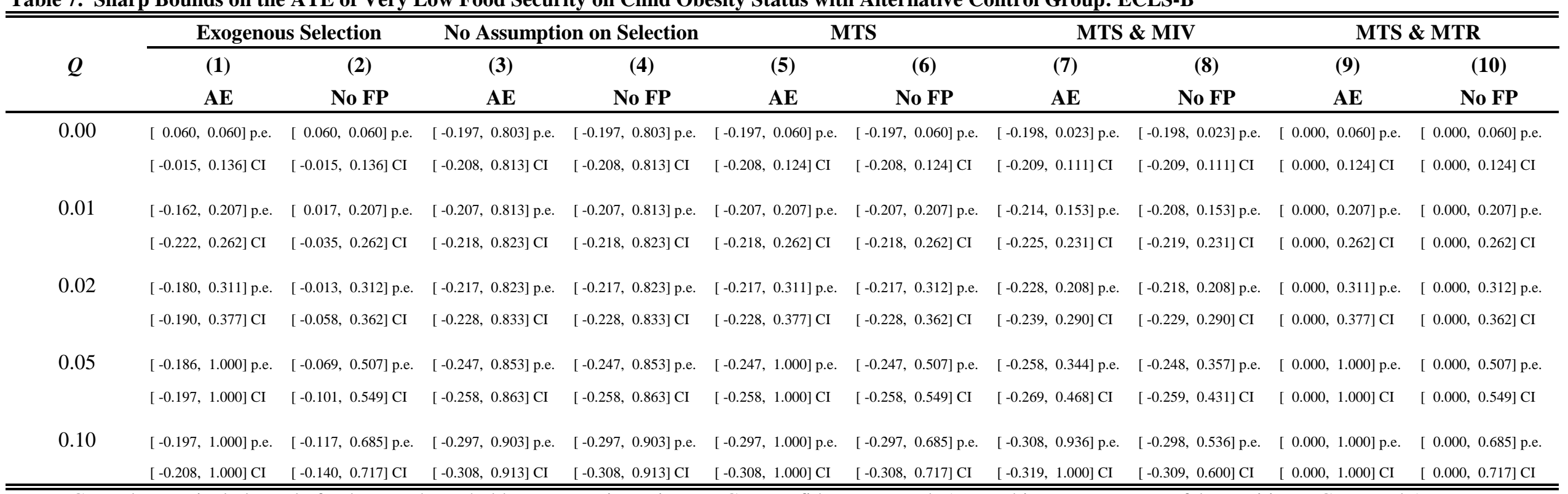

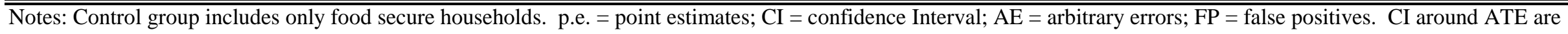

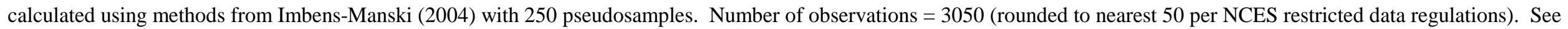
Appendix A and text for further details. 
Figure 8. Sharp Bounds on the ATE of Very Low Food Security on Child Overweight Status with Alternative Control Group: ECLS-B

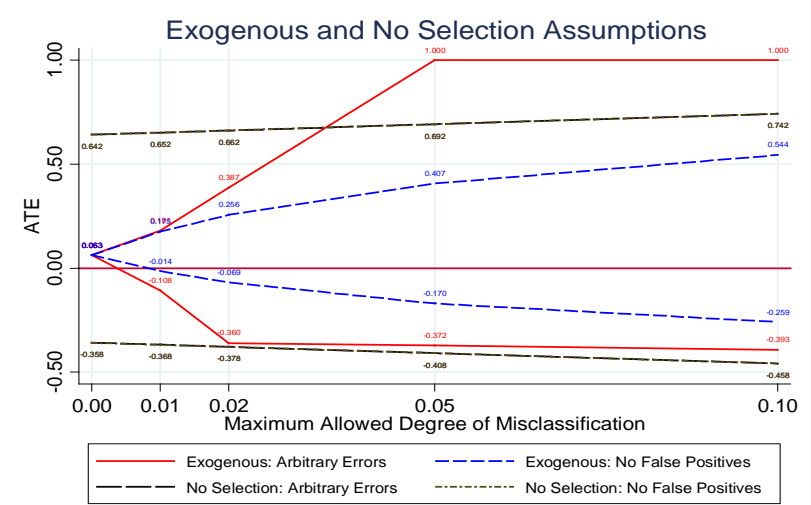

\section{A. Exogenous Selection or No Assumption}

Notes: $\mathrm{AE}=$ arbitrary errors; $\mathrm{FP}=$ false positives.

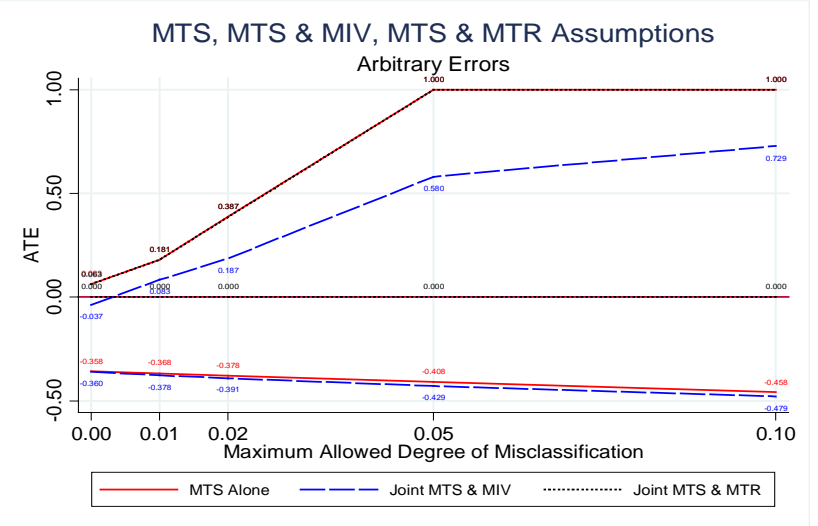

B. Only MTS or MTS-MIV or MTS-MTR: AE

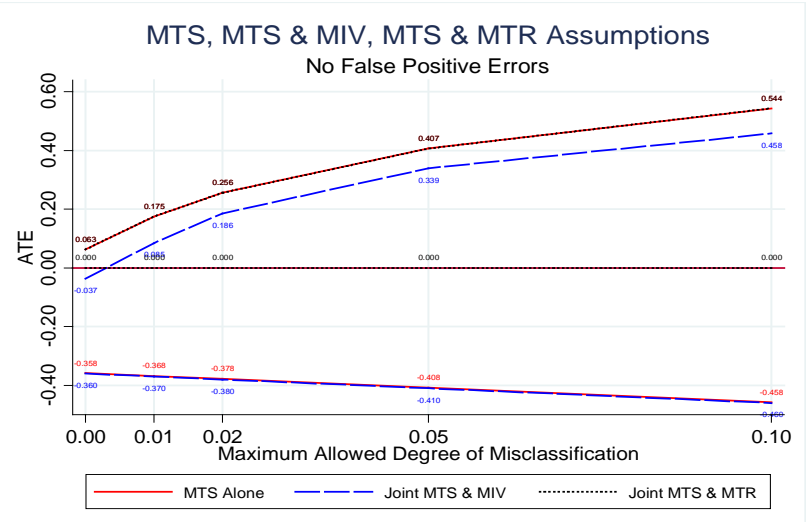

C. Only MTS or MTS-MIV or MTS-MTR: No FP

Table 8. Sharp Bounds on the ATE of Very Low Food Security on Child Overweight Status with Alternative Control Group: ECLS-B

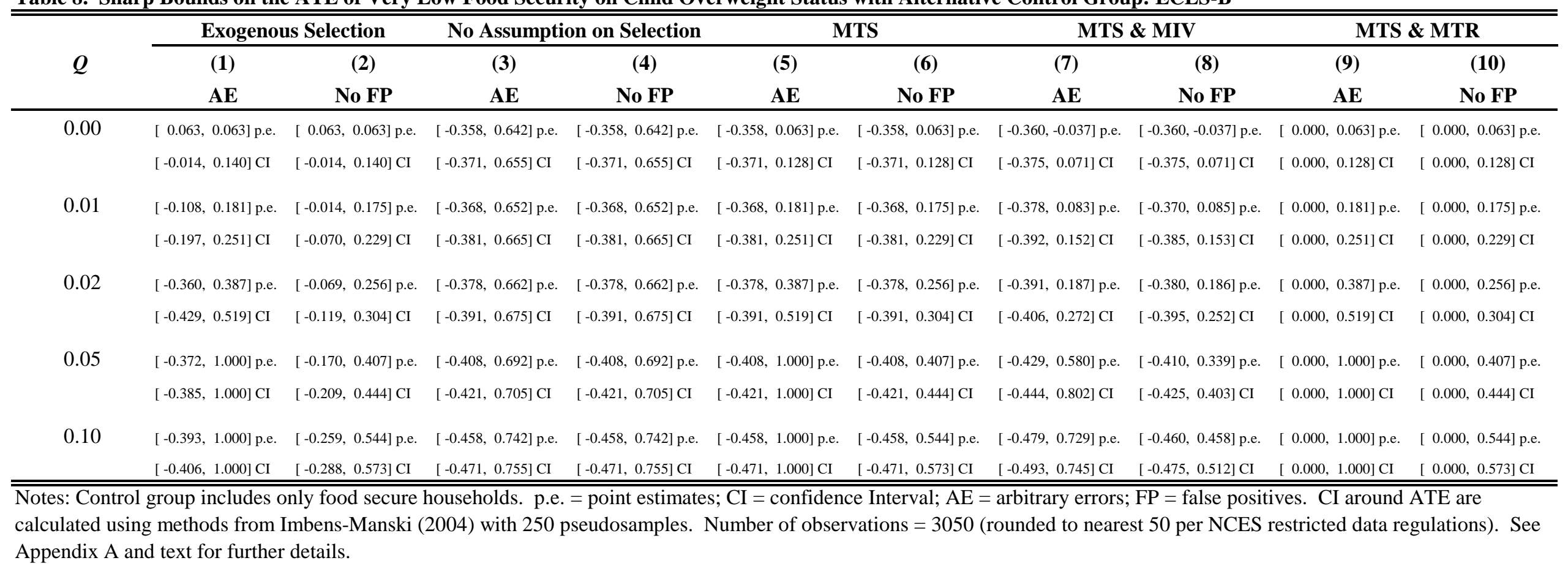

